\title{
Quantum Standing Waves and Tunneling through a Finite Range Potential
}

\author{
Haiduke Sarafian \\ University College, The Pennsylvania State University, York, USA \\ E-mail:has2@psu.edu \\ Received February 23, 2011; revised April 5, 2011; accepted April 22, 2011
}

\begin{abstract}
We consider a time independent one dimensional finite range and repulsive constant potential barrier between two impenetrable walls. For a nonrelativistic massive particle projected towards the potential with energies less than the barrier and irrespective of the spatial positioning of the potential allowing for quantum tunneling, analytically we solve the corresponding Schrodinger equation. For a set of suitable parameters utilizing Mathematica we display the wave functions along with their associated probabilities for the entire region. We investigate the sensitivity of the probability distributions as a function of the potential range and display a gallery of our analysis. We extend our analysis for bound state particles confined within constant attractive potentials.
\end{abstract}

Keywords: Quantum Tunnel Effect, Asymmetric Quantum Double-Well Potential, Quantum Standing Waves, Mathematica

\section{Introduction and Motivation}

Quantum tunneling through a finite range potential barrier is a classic quantum mechanical phenomenon. The driving interest and the thrust studying one such scenario in quantum physics is the controversial incomparable situation vs. classical physics where particles with energies less than the potential are allowed to penetrate through the barrier. A well known application of quantum tunneling is the successful explanation of the spontaneous $\alpha$ particle emission by certain unstable radioactive nuclei [1]. Examples of the tunneling effect in scientific literature are limited. The scope of the coverage of this phenomenon in standard quantum mechanics textbooks frequently are confined to one dimensional single potential barrier with initial and final free particle states [2-8]. Recent online resources [6] are also confined such that they analyze the same traditional issues addressed in [2-8]. One of the objectives of our investigation is to expand on the tunneling effect and give an extended example rarely discussed in mathematical quantum physics. In our investigation we confine our focus on one dimensional single potential barrier; however, we consider a different set of boundary conditions describing the initial and final states. Analysis of the problem yields expressing analytically the probability distributions of the particle throughout the entire region. As a secondary objective we adopt a Computer Algebra System (CAS), such as Mathematica to further our analysis [9]. Mathematica's unique capability to intertwine the numeric and graphic modules allows us to display the results of our analysis investigating the "what-if" scenarios at ease. Ironically, a text written entirely in Mathematica on Quantum Mechanics completely has ignored discussing the quantum tunneling [7].

With these objectives we craft our investigation as follows. In addition to the Introduction and Motivation, in Section 2 we present the physics and mathematics of the problem leading to analytic formulations; in this section we also address a few issues of interest such as the impact of the asymmetric potential. In order to gain physical insight, in Section 3, Numeric Analysis, utilizing Mathematica's graphic and for a set of parameters we display the wave functions along with their associated probabilities for the entire region between the two walls. In this section also we investigate the sensitivity of the wave functions as a function of the potential range. In subsection 3, we extend our search to the bound state particles confined within an attractive constant finite range potential. In this section we also analyze the impact of the asymmetric potential. We also graph the output of our analysis yielding to a gallery of displays. We 
close our work with a few concluding remarks.

\section{Fundamentals and the Physics of the Problem}

The question that we are interested to address is posed as: "Situate a constant rectangular potential barrier of value $\mathrm{V}$ and a range (thickness) $\mathrm{b}$ at a random position between two impenetrable walls separated with a distance $a+b+c$; see Figure 1. Project a massive nonrelativistic particle of mass $m$ with energies $E<V$ towards the barrier and allow quantum tunneling. Given the scenario, evaluate the probability of locating the particle between the walls".

For the assumed parameters, a non-quantum mechanical particle will bounce back and forth between the wall and the potential barrier; penetration is not allowed. However, considering the quantum mechanical phenomenon, we view the scenario from a particle-wave duality point of view. Allowing for tunneling, "intuitively", one expects to locate the "particle" somewhere between the walls including the barrier. For a particle projected along the $\mathrm{x}$-axis, the probability of locating the particle within and to the right edge of the barrier should depend on the range (thickness) of the potential. The longer the range the less chance of detecting the particle within and beyond the barrier. To quantify our expectation one needs to assume a set of parameters such as mass, energy and specification of the potential namely, $\{\mathrm{m}, \mathrm{E}, \mathrm{a}, \mathrm{b}, \mathrm{c}, \mathrm{V}\}$. Having these parameters at hand, we describe the wave functions according to Schrodinger equation.

$$
\psi^{\prime \prime}(x)+\frac{2 m}{\hbar^{2}}(E-V) \psi(x)=0
$$

Solving Equation (1) for region 1 in Figure 1 gives,

$$
\psi_{1}(x)=A_{1} e^{i k x}+B_{1} e^{-i k x}
$$

where the wave number is $k=\sqrt{\frac{2 m}{\hbar^{2}} E}$. Since the left

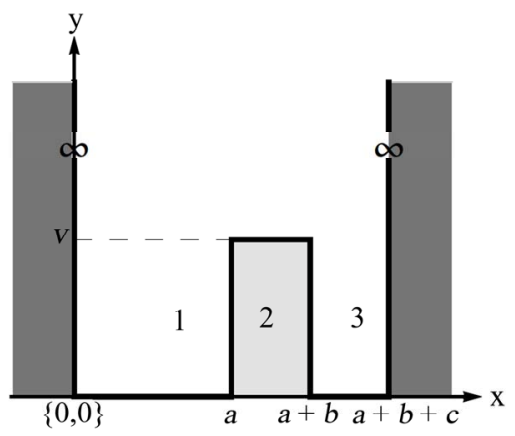

Figure 1. Display of a two-impenetrable walls separated with a distance $a+b+c$, and a constant potential barrier $V$ with thickness $b$ situated at an arbitrary distance a from one of the walls. wall at $\mathrm{x}=0$ with its $\infty$ potential requires the wave function to vanish, Equation (2) yields,

$$
\psi_{1}(x)=A \sin (k x) \text {; with } A^{*} \neq A .
$$

Solving Equation (1) for region 2 and according to the case of interest, namely, $\mathrm{E}<\mathrm{V}$ yields,

$$
\psi_{2}(x)=C e^{\kappa x}+D e^{-\kappa x}
$$

where $\kappa$ has the dimension of the inverse length and is $\kappa=\sqrt{\frac{2 \mathrm{~m}}{\hbar^{2}}(V-E)}$. Now we apply the required continuity condition across the potential boundary interface, namely, $\psi_{1}(x=a)=\psi_{2}(x=a)$. This gives,

$$
A \sin (k a)=C e^{\kappa x}+D e^{-\kappa x}
$$

Also since the slopes of the wave functions across the boundary should match, we require $\psi_{1}{ }^{\prime}(x=a)=\psi_{2}{ }^{\prime}(x=a)$. This yields,

$$
A k \cos (k a)=\kappa\left(C e^{\kappa a}-D e^{-\kappa a}\right)
$$

Manipulating Equations (5) and (6) gives,

$$
\mathrm{D}=\mathrm{Ce}^{2 \kappa \mathrm{a}}\left[\frac{\tan (k a)-\frac{k}{\kappa}}{\tan (k a)+\frac{k}{\kappa}}\right]
$$

Furthermore manipulating Equations (5) and (7) yields,

$$
C=A\left\{\frac{1}{2} e^{-\kappa a}\left[\sin (k a)+\frac{k}{\kappa} \cos (k a)\right]\right\}
$$

Similarly utilizing Equations (7) and (8) yields,

$$
D=A\left\{\frac{1}{2} e^{\kappa a}\left[\sin (k a)-\frac{k}{\kappa} \cos (k a)\right]\right\}
$$

Now we focus on region 3 shown in Figure 1. The wave function is,

$$
\psi_{3}(x)=E_{3} e^{i k x}+F_{3} e^{-i k x}
$$

Here again we set $\psi_{3}(x=a+b+c)=0$; this gives $E_{3}=-F_{3} e^{-2 i k(a+b+c)}$. Utilizing Equation (10) yields,

$$
\psi_{3}(x)=F \sin [k(a+b+c-x)] \text { with } \mathrm{F}^{*} \neq \mathrm{F}
$$

Matching the wave functions across the right edge of the potential requires, $\psi_{2}(x=a+b)=\psi_{3}(x=a+b)$. This is,

$$
C e^{\kappa(a+b)}+D e^{-\kappa(a+b)}=F \sin (k c)
$$

Combining Equations (8), (9) and (12) gives,

$$
\begin{aligned}
& F= \\
& A\left\{\frac{1}{\sin (k c)}\left[\sin (k a) \cosh (\kappa b)+\frac{k}{\kappa} \cos (k a) \sinh (\kappa b)\right]\right\}
\end{aligned}
$$


In short,

$$
\begin{aligned}
& \psi(x)= \\
& \left\{\begin{array}{l}
\psi_{1}(x)=A \sin (k x) ; 0 \leq x \leq a \\
\psi_{2}(x)=C(A) e^{\kappa x}+D(A) e^{-\kappa x} ; a \leq x \leq a+b \\
\psi_{3}(x)= \\
F(A) \sin [k(a+b+c-x)] ; a+b \leq x \leq a+b+c
\end{array}\right.
\end{aligned}
$$

where $\mathrm{C}(\mathrm{A}), \mathrm{D}(\mathrm{A})$ and $\mathrm{F}(\mathrm{A})$ are given by Equations (8), (9) and (13).

On the other hand the wave function $\psi(x)$ is normalized throughout the entire region. In other words,

$$
\int_{0}^{a+b+c}|\psi(x)|^{2} d x=1
$$

Substituting for the wave functions according to Equation (14) yields the value of the amplitude, A,

$$
A=\frac{1}{\sqrt{i_{1}+i_{2}+i_{3}}}
$$

where $\left\{i_{1}, i_{2}, i_{3}\right\}$ are,

$$
\left\{\begin{array}{l}
i_{1} \rightarrow \frac{1}{2}\left[a-\frac{1}{2 k} \sin (2 k a)\right] \\
i_{2} \rightarrow\left(\frac{C(A)}{A}\right)^{2} \frac{1}{2 \kappa}\left(e^{2 \kappa(a+b)}-e^{2 \kappa a}\right)-\left(\frac{D(A)}{A}\right)^{2} \\
\frac{1}{2 \kappa}\left(e^{-2 \kappa(a+b)}-e^{-2 \kappa a}\right)+2 C(A) D(A) \\
i_{3} \rightarrow \frac{1}{2}\left[\frac{\sin (k a)}{\sin (k c)} \cosh (\kappa b)+\frac{k}{\kappa} \frac{\cos (k a)}{\sin (k c)} \sinh (\kappa b)\right]^{2} \\
{\left[c-\frac{1}{2 k} \sin (2 k c)\right]}
\end{array}\right.
$$

Thus far we have addressed the coefficient issue; however, we may further our investigation. Matching the slopes of the wave functions across the right edge of the potential requires, $\psi_{2}{ }^{\prime}(x=a+b)=\psi_{3}{ }^{\prime}(x=a+b)$. Implementing the latter along with Equation (12) gives,

$$
D=C e^{2 \kappa(a+b)}\left[\frac{\tan (k c)-\frac{k}{\kappa}}{\tan (k c)+\frac{k}{\kappa}}\right]
$$

And finally from Equations (7) and (16) we get,

$$
\begin{aligned}
& \left(\frac{k}{\kappa}\right)^{2}+[\tan (k a)+\tan (k c)] \operatorname{coth}(\kappa b) \frac{k}{\kappa}+ \\
& \tan (k a) \tan (k c)=0
\end{aligned}
$$

Equation (17) is an equation free of amplitudes. It im- plicitly intertwines the relevant geometric parameters of the potential, namely $\{a, b, c\}$ to the energy of the particle, $\mathrm{E}$ and to the strength of the potential, V. Equation (17) may also be arranged as,

$$
\tanh (\kappa b)+\frac{k}{\kappa}\left[\frac{\tan (k a)+\tan (k c)}{\tan (k a) \tan (k c)+\left(\frac{k}{\kappa}\right)^{2}}\right]=0
$$

Simply put, utilizing either Equation (17) or (18) evaluates the permissible, "allowed" energies, E, compatible with the rest of the parameters. At a first glance it may appear that Equation (18) which is a manifestation of the Equation (17) provides the same information. However, in Section 3 we'll prove otherwise.

Literature search reveals a simplified version of the proposed problem for a symmetrically positioned potential, namely $\mathrm{c}=\mathrm{a}$, has been somewhat analyzed [10]. However, the analysis of the latter is purely numeric and the author's objectives of those analyses deviate from our own. Our analysis is purely analytical and the detailed steps are conducive to final explicit results. In other words, having the symbolic generalized formulation at hand allows us to analytically investigate symmetric cases as well. In short, setting $\mathrm{c}=\mathrm{a}$ in Equation (14) gives the wave functions and the needed coefficients for the symmetrical potential; these are missing in [10].

\section{Numeric Analysis}

This section is composed of three subsections. The first subsection deals with the symmetric potential barrier. The second subsection deals with an asymmetrically positioned barrier and the last subsection is the presentation of the information in regards to the bound state attractive constant potential.

\subsection{Case 1, Symmetric Positive Potential}

In this subsection we consider a symmetrically positioned potential barrier. We set $c=a$; this positions the barrier symmetrically between the two walls. The equation describing the wave function in region 3, i.e. $\psi_{3}(x)$, changes accordingly. The last row of Equation (14) becomes $\psi_{3}(x)=F(A) \sin [k(2 a+b-x)]$ and its amplitude according to Equation (13) changes to

$$
F(A)=A\left[\cosh (\kappa a)+\frac{k}{\kappa} \cot (k a) \sinh (\kappa b)\right]
$$

Also the quantity $i_{3}$ given in Equation (15) changes appropriately. With this wave function at hand we consider reasonable parameters to evaluate the probability distributions. We consider an electron, $m \cdot c^{2}=0.5 \mathrm{MeV}$, and for the geometric parameters we take $\{a, b, c\}=\{4,2,4\}$ 
angstrom, and we set $V=10 \mathrm{eV}$. Substituting for $\hbar c=197$ Mev fm, the wave number $k$ and the quantity $\kappa$ in the units of $1 / \AA$ become,

$$
\begin{aligned}
& \{k, \kappa\}=\left\{\frac{100 .}{\hbar c} \sqrt{2 m c^{2}} \sqrt{E}, \frac{100 .}{\hbar c} \sqrt{2 m c^{2}} \sqrt{V-E}\right\}= \\
& \{0.50764 \sqrt{E}, 0.50764 \sqrt{V-E}\}
\end{aligned}
$$

In these quantities the energy, $E$, is to be entered in $\mathrm{eV}$ units.

The chosen set of parameters is intertwined via either Equation (17) or Equation (18). To evaluate the permissible values of energy $E$ one may graph the left hand side of Equation (18) and hunt for compatible energies within the range $0<E<V$. Alternatively one may plot Equation (17) and look for its intersecting coordinates with the $\mathrm{E}$ axis. Equation (18), however, is a second order algebraic equation with two distinct roots. Therefore, we solve Equation (17) and plot functions associated with its roots along with the plot of the left hand side of Equation (18). This gives two separate graphs shown in Figure 2.

This analysis is critical for appropriately grouping the roots of the equations. Without this analysis one may apply the unsorted roots of Equation (18) achieving unsightly conclusions. Overlaid plots of Equation (18) along with the plots of the root equations of Equation (17) provide the natural sorting. The red curve of top graph of Figure 2 is the positive root of Equation (17) while the green curve is the plot of Equation (18). This clearly shows the red curve intersects with the smaller values of the paired green intercepts. On the contrary, as shown on the bottom graph the plot of the other root of Equation (17) intersects at the larger values of the paired green intercepts. In short, with this learned insight one would be better off seeking the roots of Equation (17) separately rather than seeking the roots of Equation (18). Alternatively, if one chooses to utilize Equation (18) one would need to be cautious sorting the roots conducive the same previous conclusions. Having said all this, now we pursue evaluating the sorted roots of Equation (17). These are tabulated in Table 1.

In Table 1, the first row, "Plus", corresponds to the positive roots of Equation (17), and the second row, "Minus", is the corresponding roots of the negative root equation. With these values of permissible energies we plot the wave functions and their associated probability distributions; these are shown in Figure 3. There are two permissible energies; their ordered list is $n=\{1,2\}$. It is notable that the lower energies of each set are associated with the even wave functions. These are the first plots of graphs of each row. The wave functions are symmetrical about the center of the potential barrier. For a visual understanding three different colors are used to show the wave functions in different regions. The continuity of the
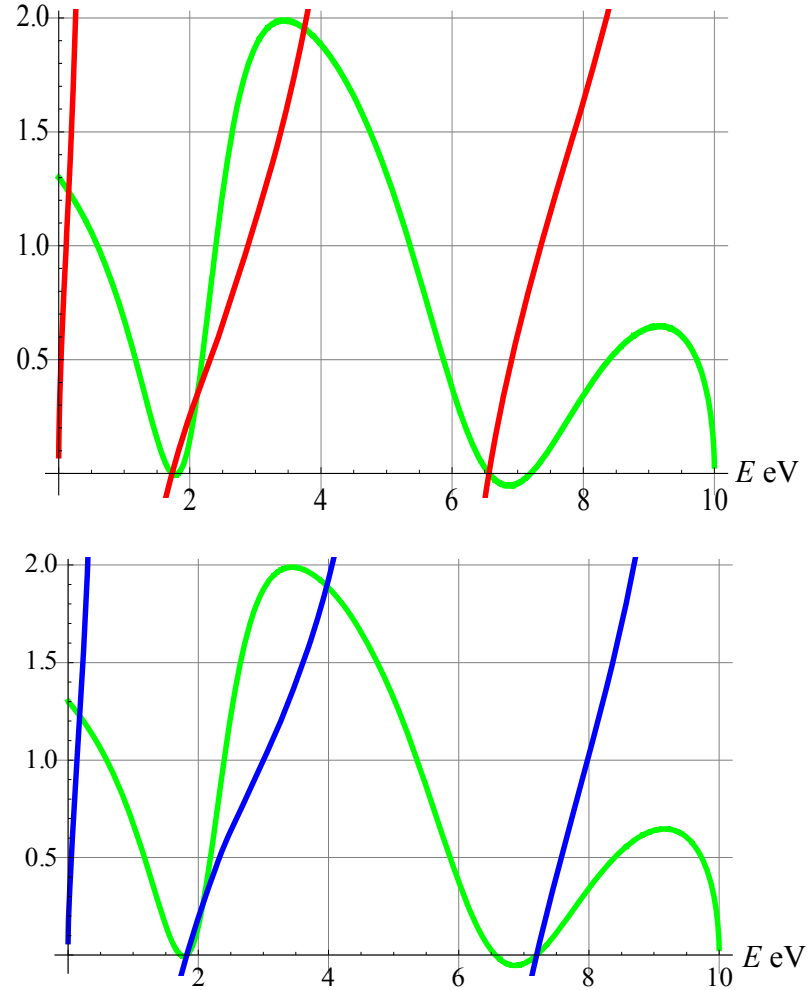

Figure 2. The green curve is the plot of left hand side of Equation (18). The red curve on the left graph is the plot of the positive root of Equation (17), while the blue curve of the right plot is the display of the negative root of Equation (17).

Table 1. The entries in the first row are the roots of Equation (17) associated with its positive root. The second row is the repeat of the same scenario for the negative roots.

\begin{tabular}{ccc}
\hline & Root 1 & Root 2 \\
\hline Plus & 1.72966 & 6.56287 \\
Minus & 1.82285 & 7.19653
\end{tabular}

wave functions across the edges of the potential barrier clearly is depicted. The wave functions associated with the higher permissible energy set are depicted in the middle column. These wave functions are asymmetric and three different colors are used to show the associated wave functions in their respective regions. The author is pleased to point out that color coding concept of the regional wave functions is novel and unique to this project.

As required the wave functions are clamped at both ends. The lowest permissible energy is associated with the simplest configuration of the even wave function and is represented by the top graph. The latter wave function is a symmetrical function with respect to the center of the potential barrier and it does not cross the horizontal axis. On the contrary, the energy of the odd wave function is higher than the corresponding even wave function and it 
crosses the horizontal axis; the odd wave function is represented by the middle graph. The same observations are true for the graphs on the second row.

Utilizing the wave functions displayed in Figure 3 we display also the probability distribution associated with each case; these distributions are depicted in Figure 4. One notices that the probability distributions are distin- guishable only for the higher permissible energies associated with $\mathrm{n}=2$.

To form a comprehensible opinion about the relationship of the probability distributions and their associated potential barrier, selectively in Figure 5 we display the potential barrier and the probability function for one case,
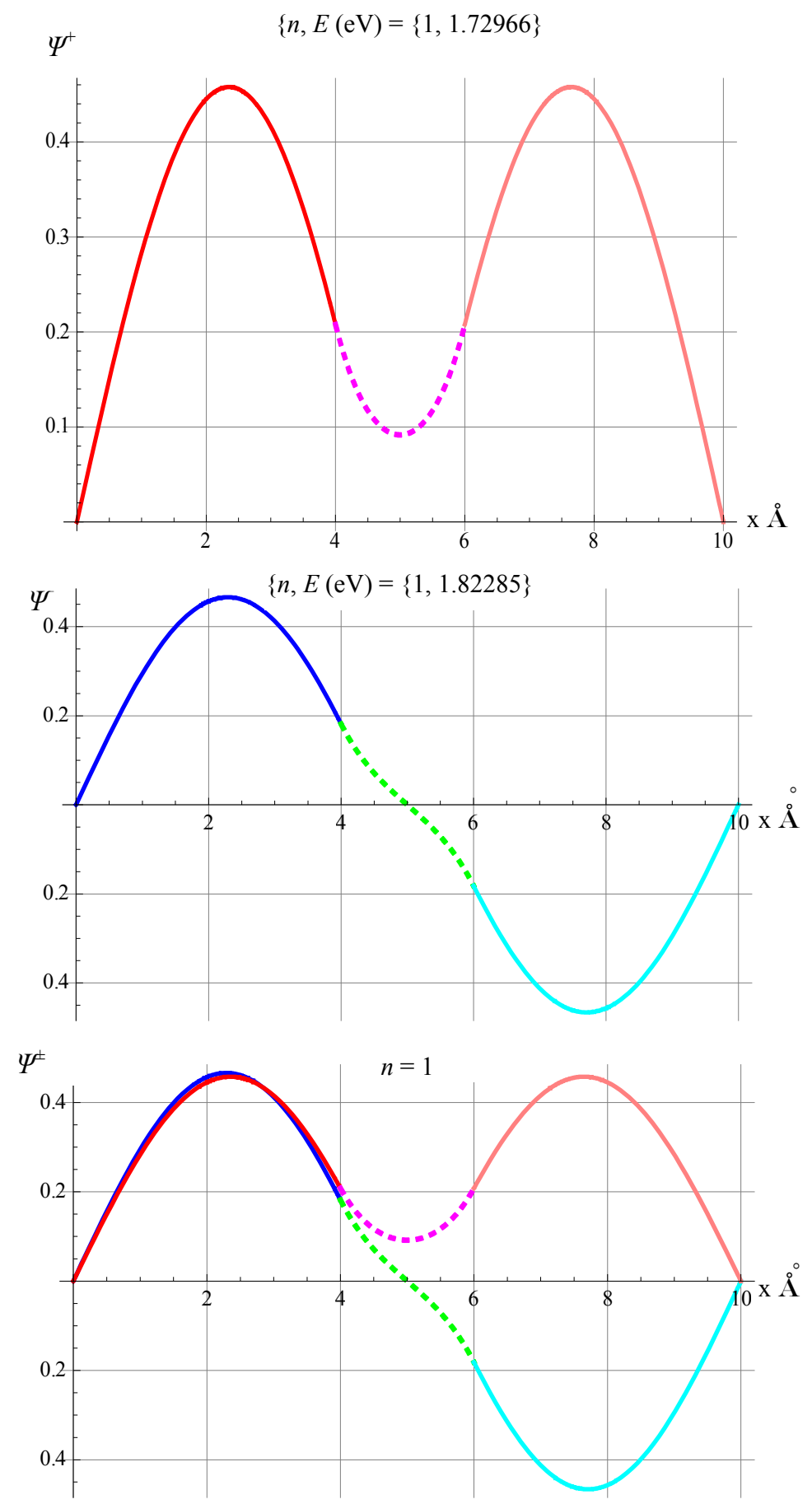

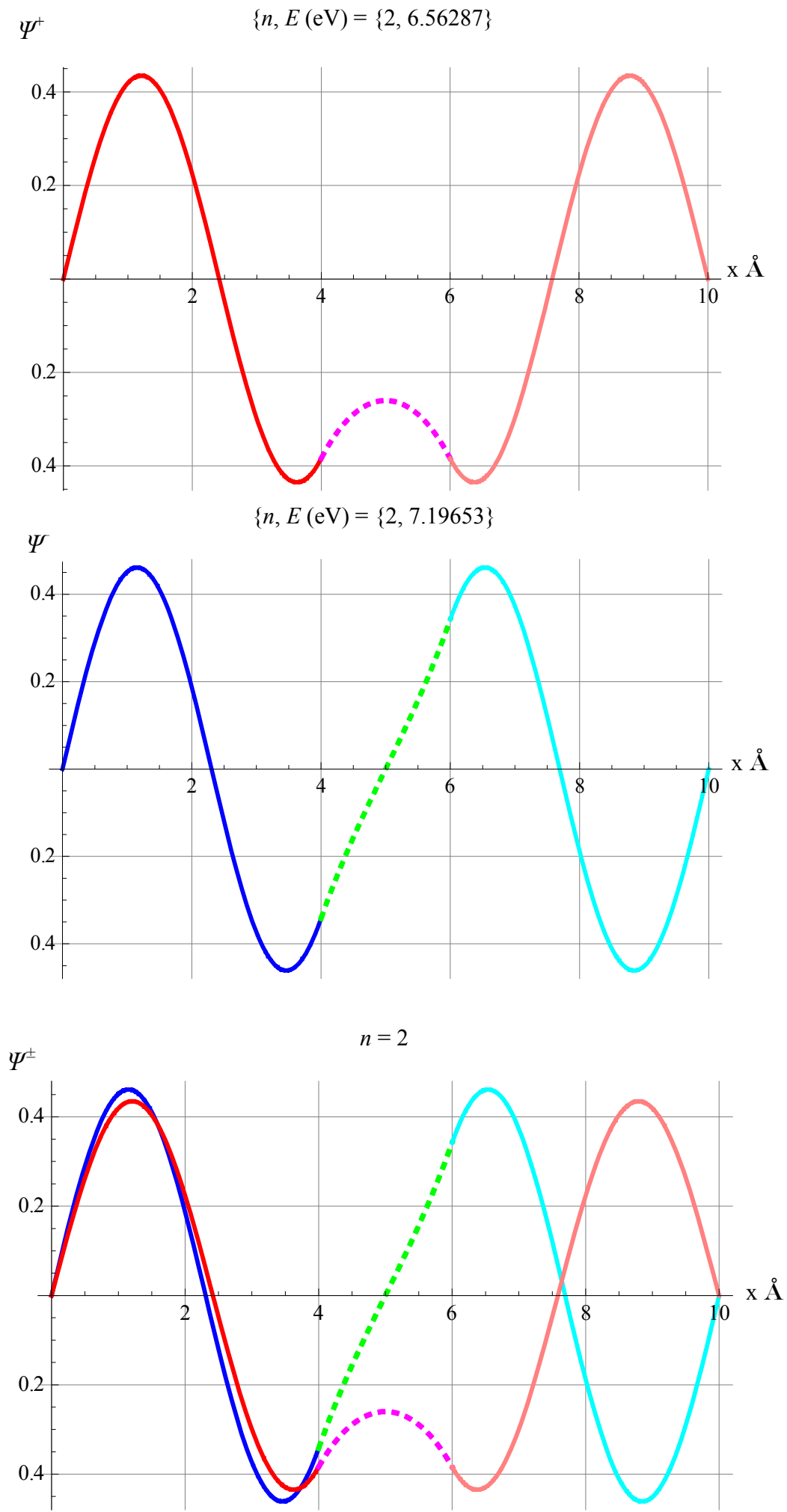

Figure 3. From top to bottom, the first row is the even wave function associated with the lower permissible energy. The second row is the odd wave function associate with the higher permissible energy. The third row is the overlay of the first and the second rows. The parameters of the potential are $\{a, b, c\}=\{4,2,4\}$. 
e.g. $n=2$.

We further our search by analyzing the sensitivity of the wave functions as a function of the thickness, or scientifically speaking, the range of the constant potential. For three cases namely for thicknesses $b=4,1$, and 0.01 ang- strom we display the respective wave functions. At the outset one knows that altering the parameters of the problem, namely the thickness $b$ would require solving the Equation (17) and/or (18) all over again. For instance, and for the sake of guidance we provide Table 2. This table
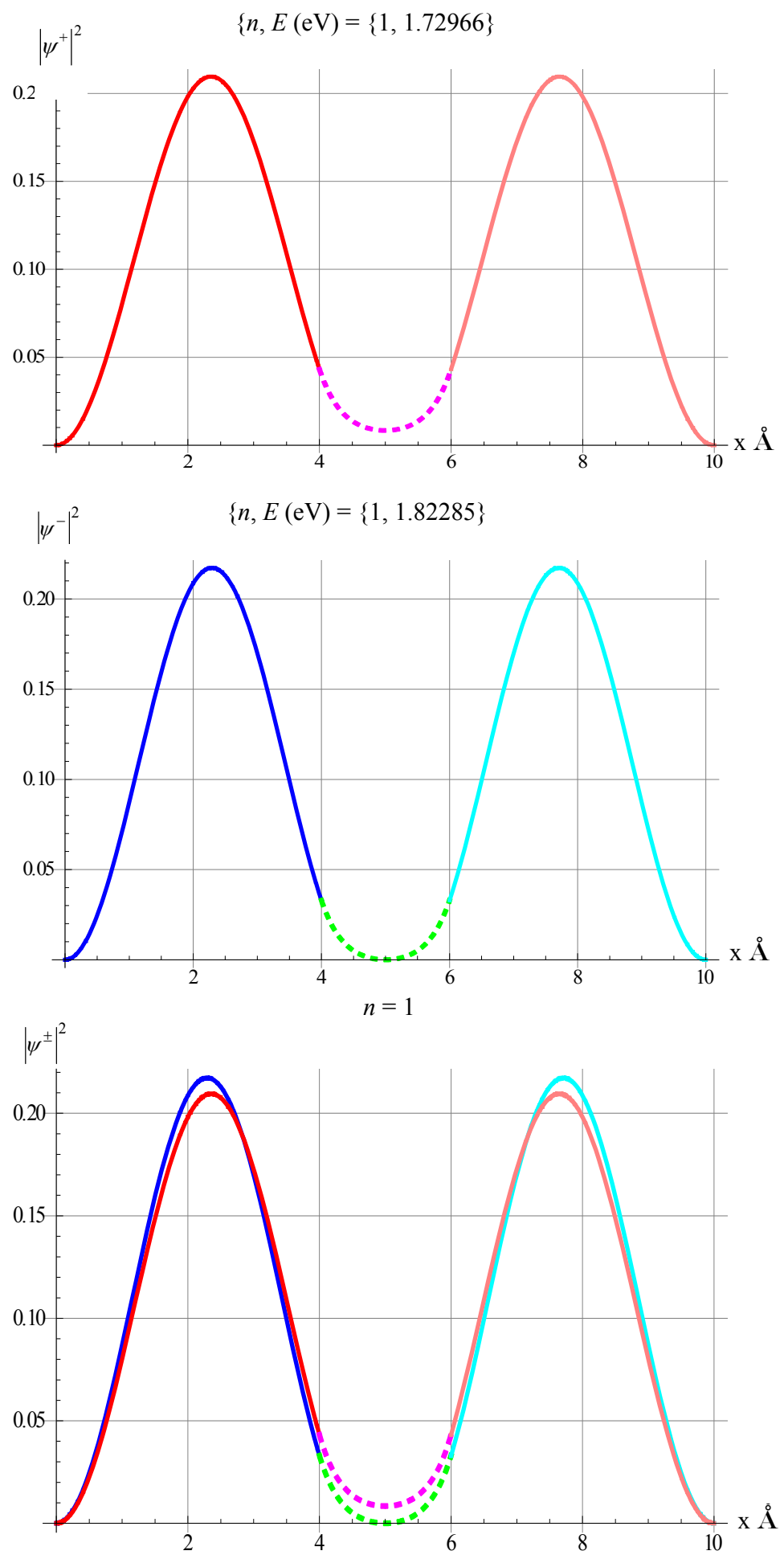

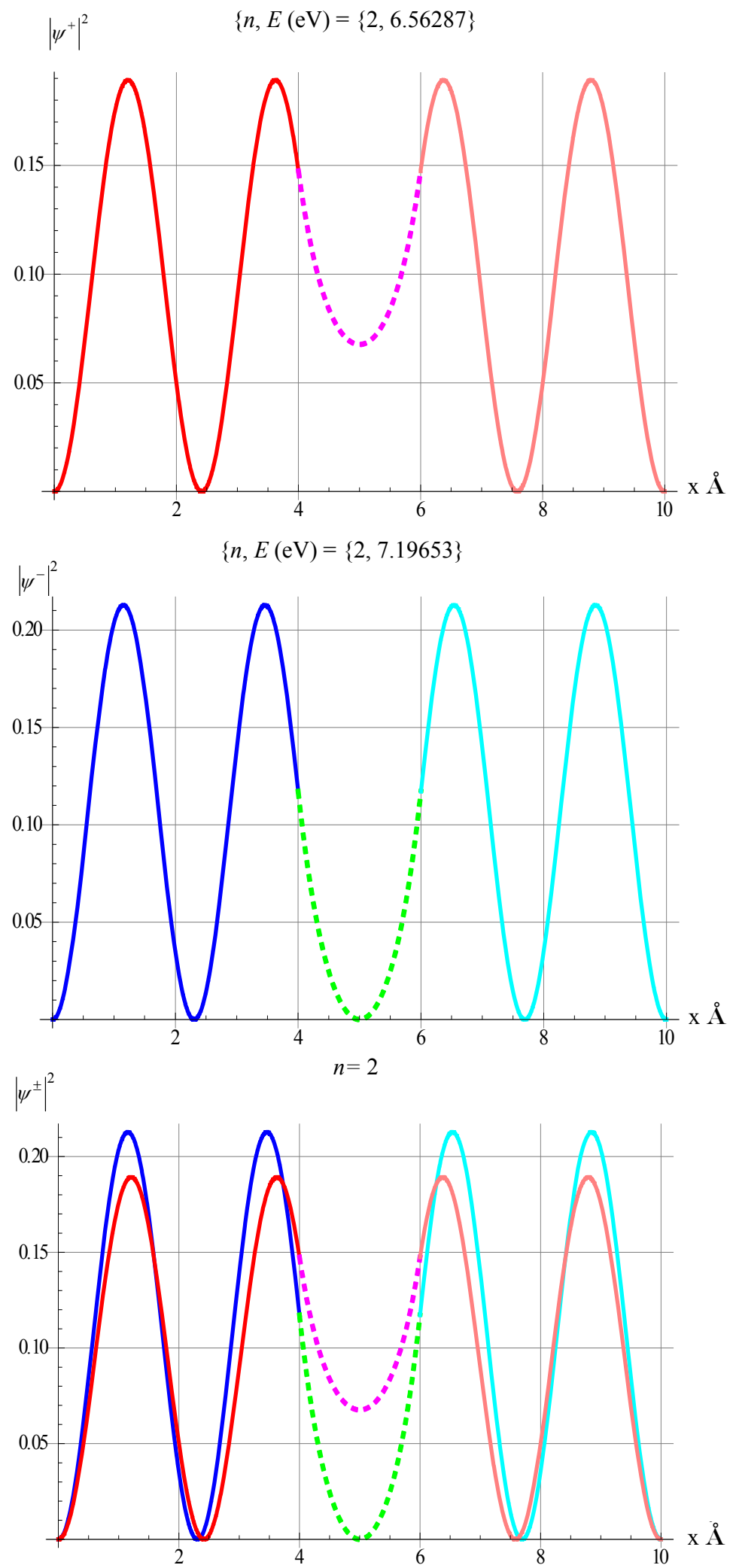

Figure 4. From top to bottom, the first row is the even wave function associated with the lower permissible energy. The second row is the odd wave function associate with the higher permissible energy. The third row is the overlay of the first and the second rows. 

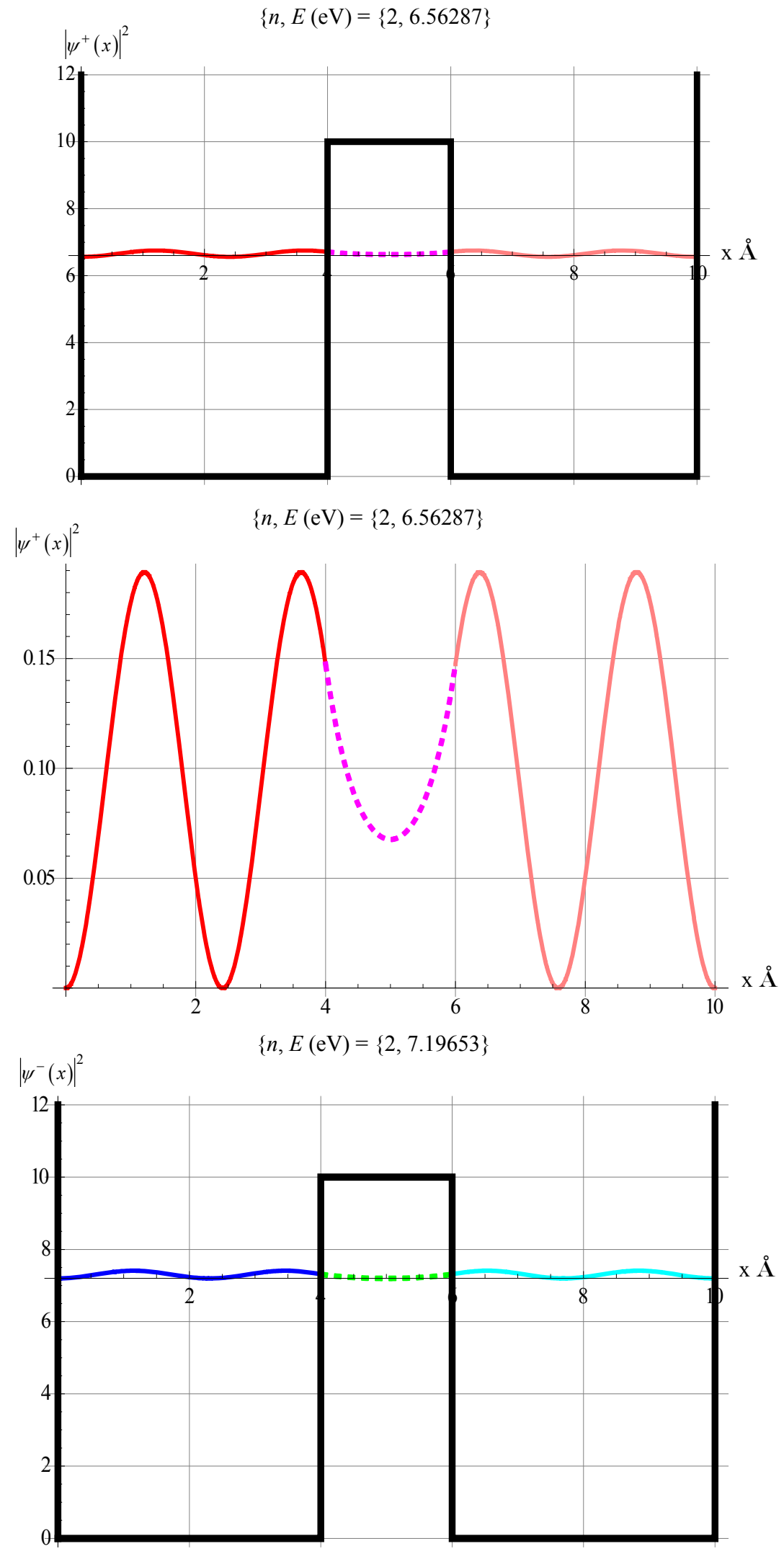


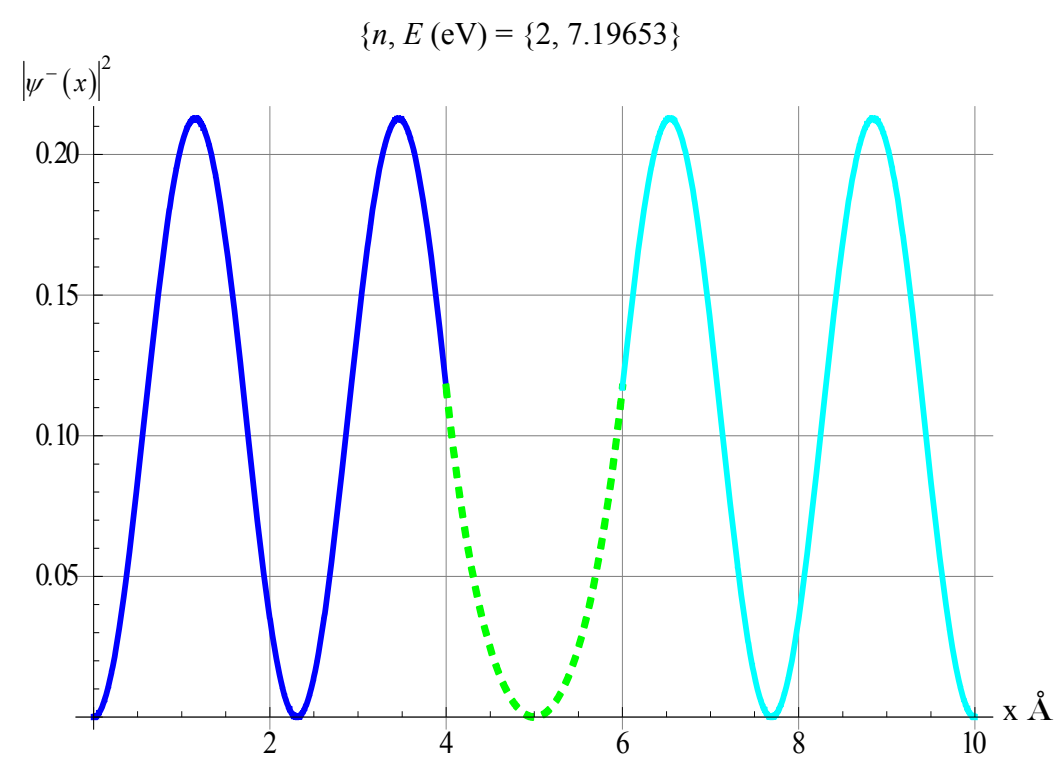

Figure 5. Display of the potential barrier and the probability distributions for $n=2$. The first row of graphs corresponds to the even wave function, and the second row of graphs corresponds to the odd wave function.

Table 2. This table is similar to Table 1 , and corresponds to the roots of Equation (17) for the barrier thickness $b=4 \AA$.

\begin{tabular}{ccc}
\hline & Root 1 & Root 2 \\
\hline Plus & 1.7742 & 6.78903 \\
Minus & 1.77927 & 6.89066 \\
\hline
\end{tabular}

contains the roots of Equation (17) for $b=4$.

The general features of the wave functions displayed in Figure 6 are somewhat the same as the corresponding wave functions in Figure 3. Noticeably, the wider potential hampers the tunneling effect, reducing the probability of locating the particle in the 2 nd region.

The next two set of graphs are associated with the $\mathrm{b}=1.0$ and $0.01 \AA$, respectively.

Here again the general features of the wave functions are somewhat similar to the corresponding graphs of Figure 3. Noticeably, the narrower potential enhances the tunneling, promoting the probability of locating the particle.

As expected a very narrow potential barrier totally ignores the tunneling. The continuity of the wave function for such a potential naturally is enforced.

Analyzing these sets of plots shown in Figures 3, 4, 6, and 7 reveals that irrespective of the thickness of the barrier, the continuity of the wave functions are preserved. As a general observation we realize depending on the values of the permissible energy the number of the crossings of the wave functions with the $\mathrm{x}$-axis increases; the higher the energy the larger the number of crossings. Also it appears the plots of Figure 8 are the only ones that are intuitive. These plots are corresponding to an almost zero barrier thickness and directly connect the wave functions of region 1 to region 3 .

\subsection{Case 2, Asymmetric Positive Potential}

In this subsection we consider an asymmetrical potential. Positioning the potential barrier asymmetrically breaks the symmetry of the wave functions. However, it is not intuitive what to expect. To show the impact of the asymmetric potential we consider a case such as, $\{a, b, c\}$ $=\{4,2,6\}$. Comparing the parameters of this case to the parameters of the compatible symmetrical potential namely $\{a, b, c\}=\{4,2,4\}$ one naively expects minor differences. However, solving Equation (17) provides a set of six paired roots; these are entered in Table 3. As shown earlier, each set of energy corresponds to a different set of even and odd wave functions. Therefore, according to Table 3 there are six sets of wave functions. However, for the sake of managing the length of our manuscript we display only one set of wave functions; these are corresponding to $\mathrm{n}=6$.

Here again for the asymmetrical potential we notice the wave functions are continuous across the potential barrier. The color codes facilitate our ability to relate the wave functions to the corresponding regions. The second column of plots is the magnified version of the same wave functions plotted in the first column. The last row is the cap stone graph; this compares the "even" and the "odd" wave functions. The author patiently also analyzed the sensitivity of the wave functions as a function of various geometric parameters of the potential. These are not reported here, but are compiled in a lengthy graphic atlas. 
H. SARAFIAN
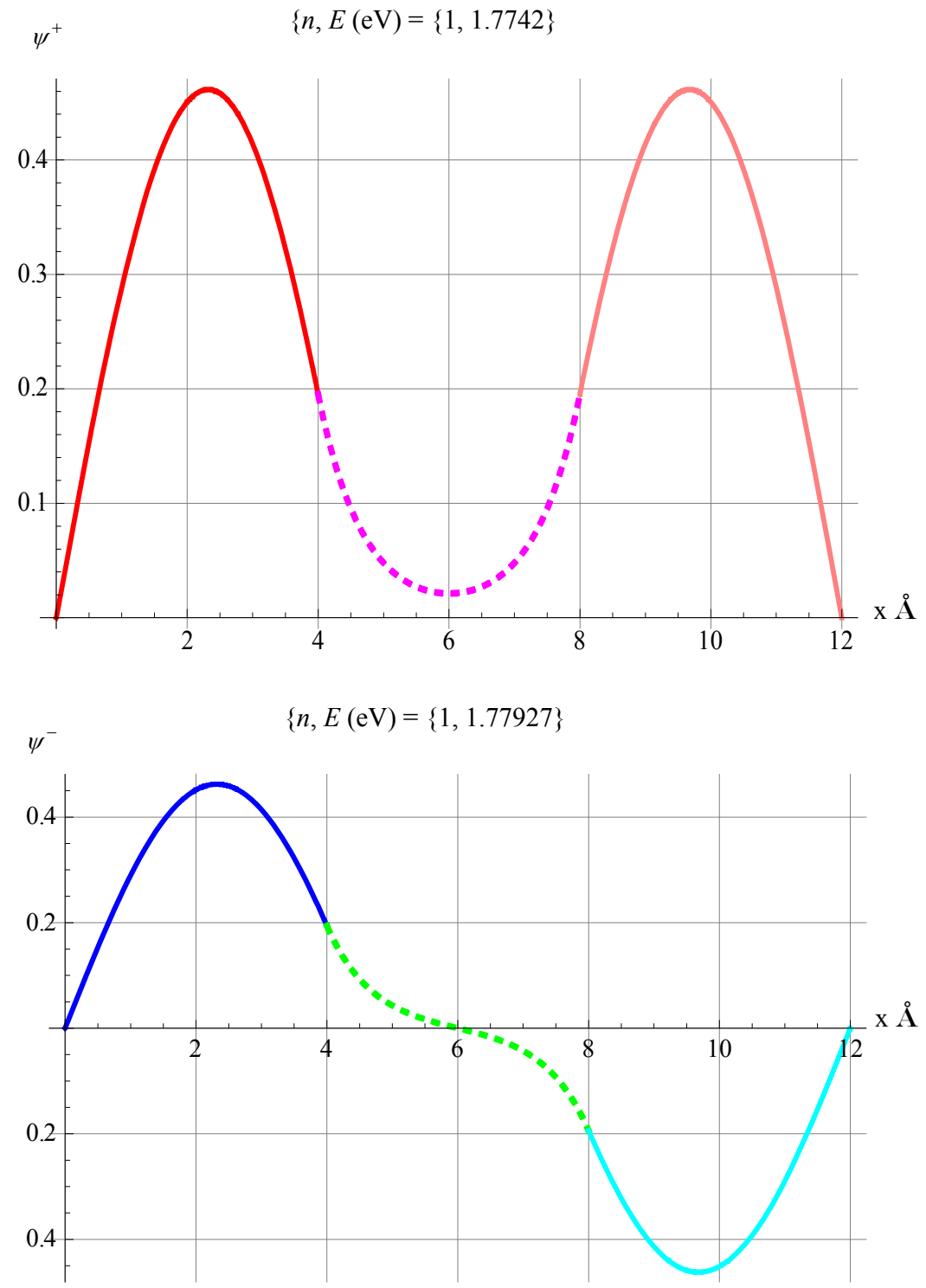

$n=1$

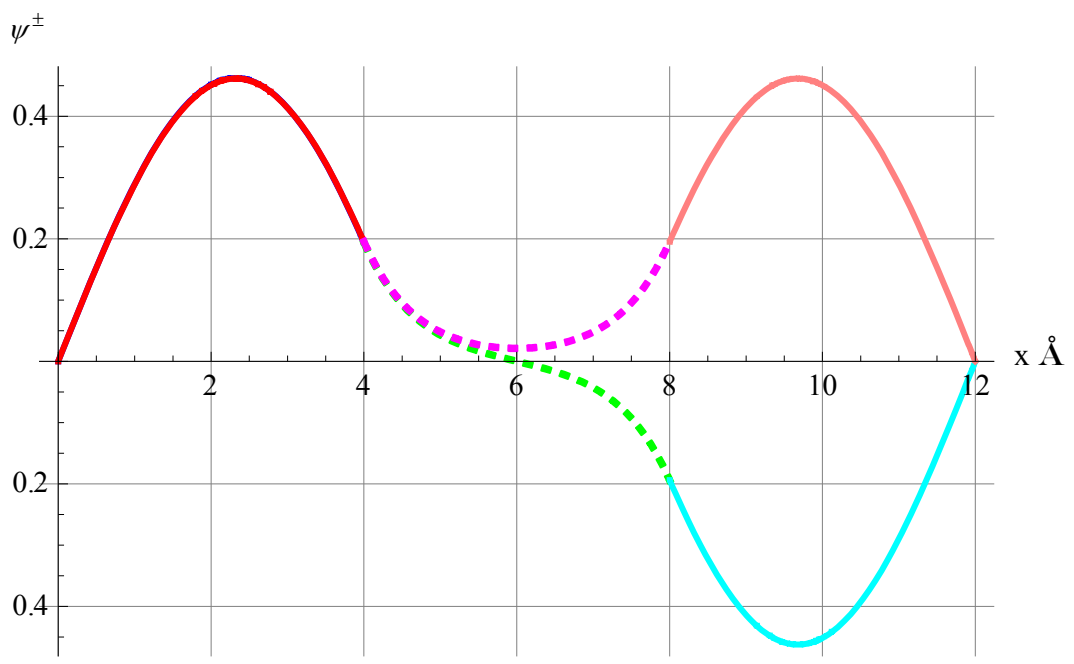

Copyright (C) 2011 SciRes.

JMP 
686

H. SARAFIAN
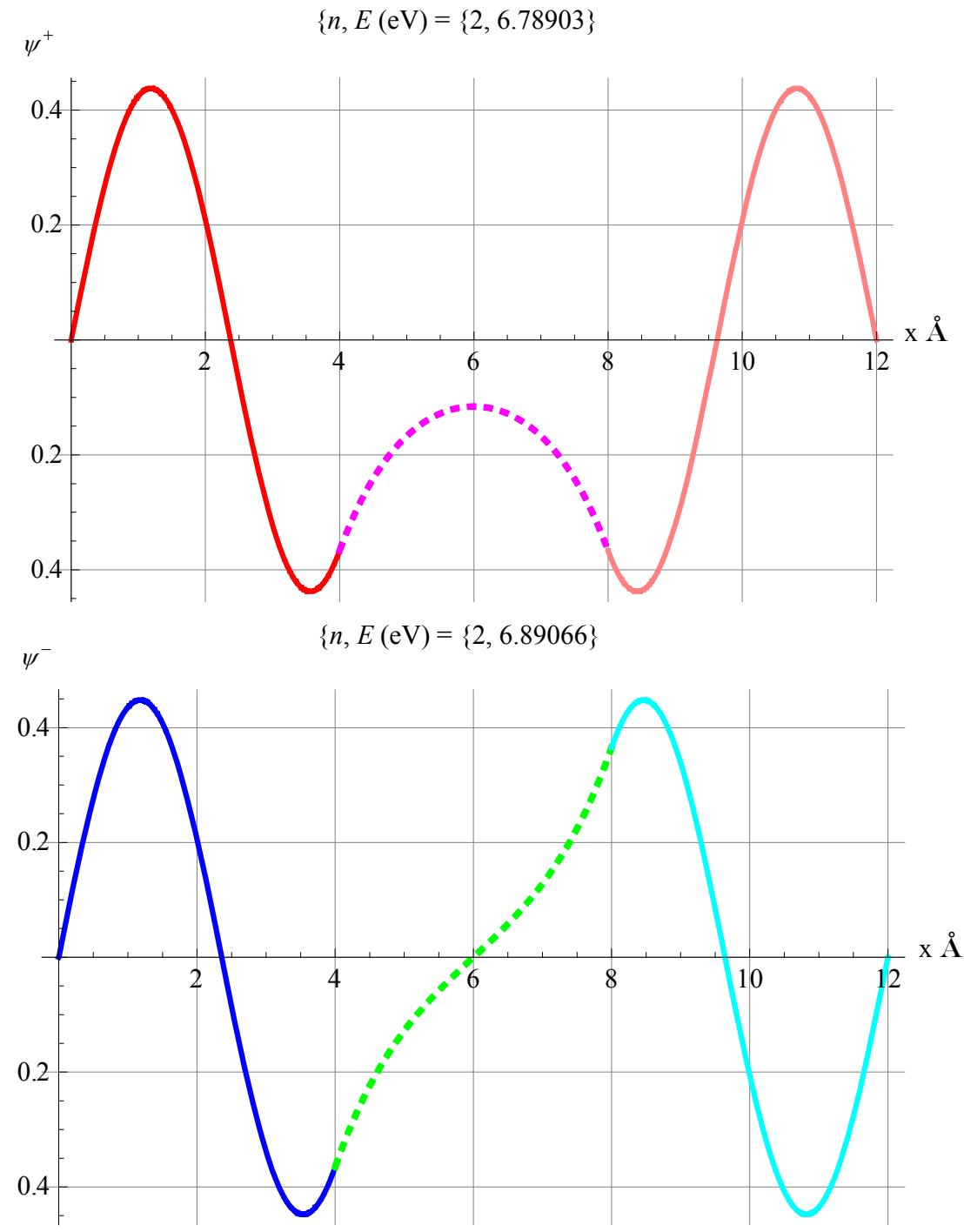

$$
n=2
$$

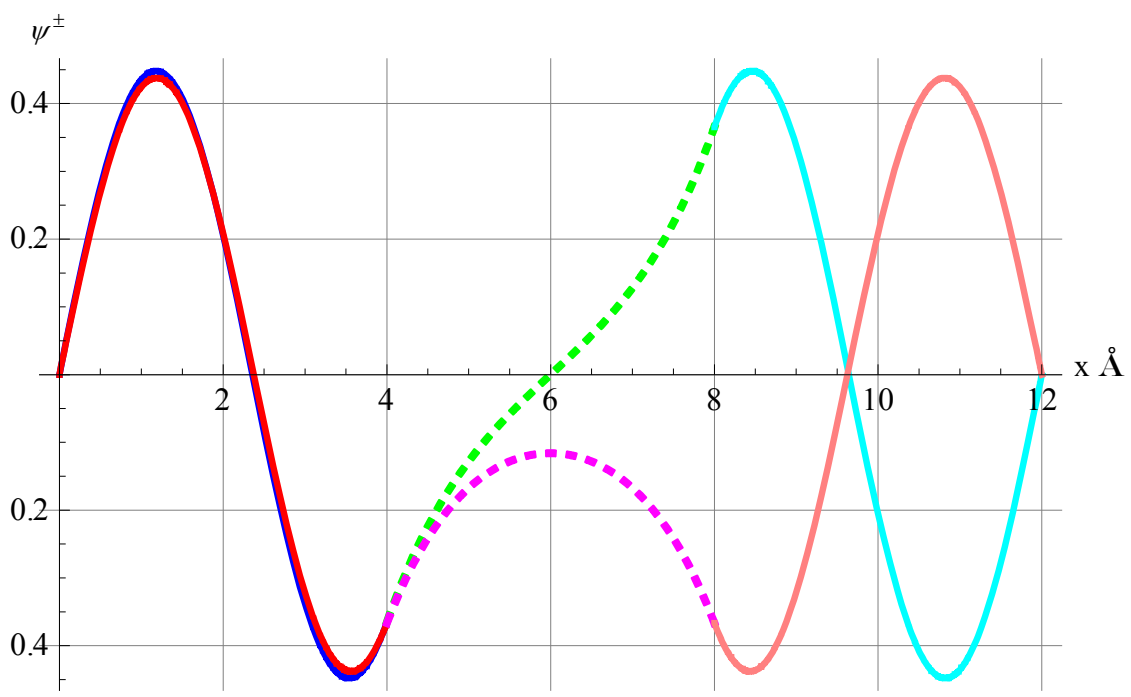

Figure 6. Description of these figures is the same as Figure 3. In this figure, the graphs are associated with $b=4.0 \AA$.

Copyright (C) 2011 SiRes.

IMP 
H. SARAFIAN
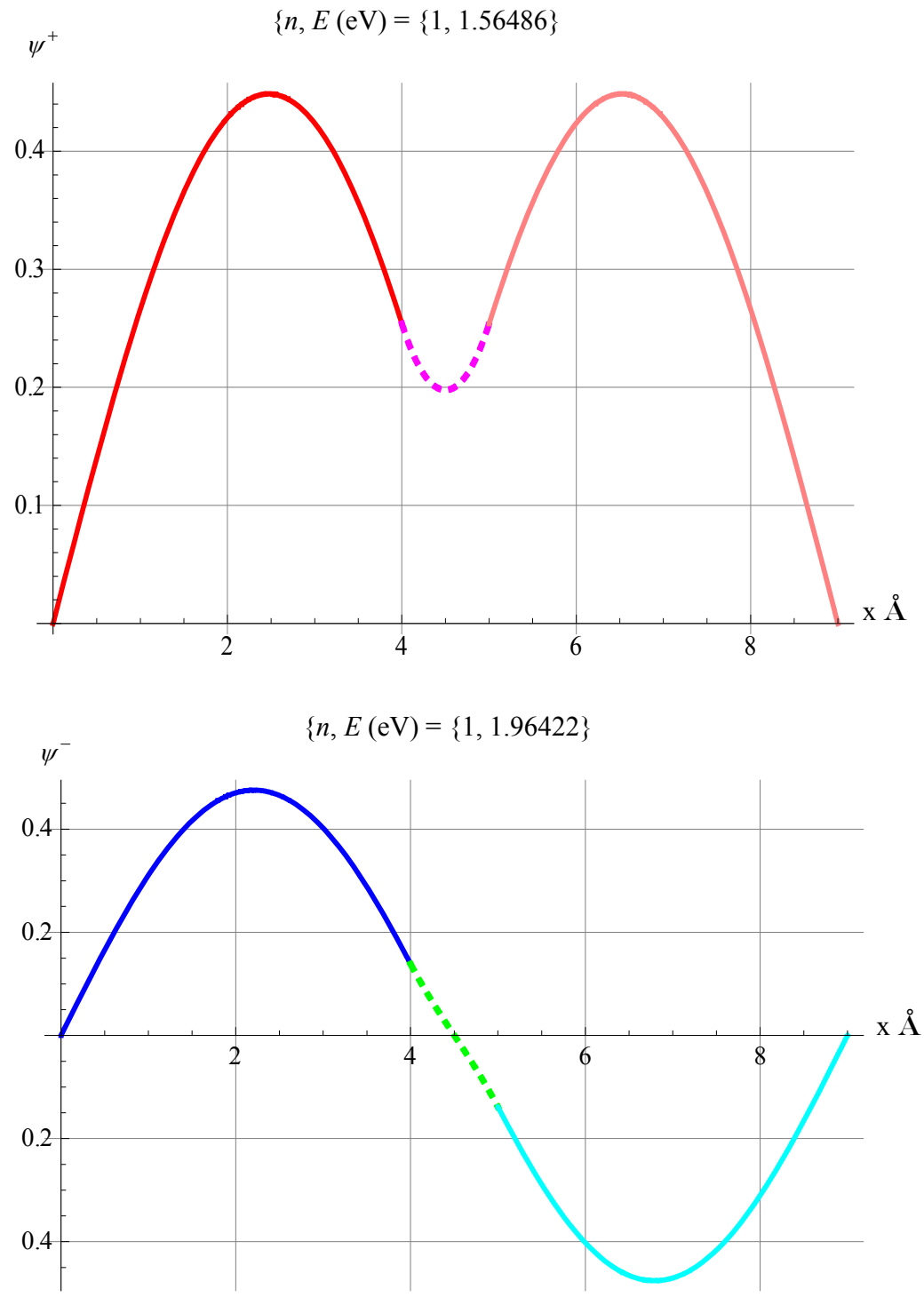

$n=1$

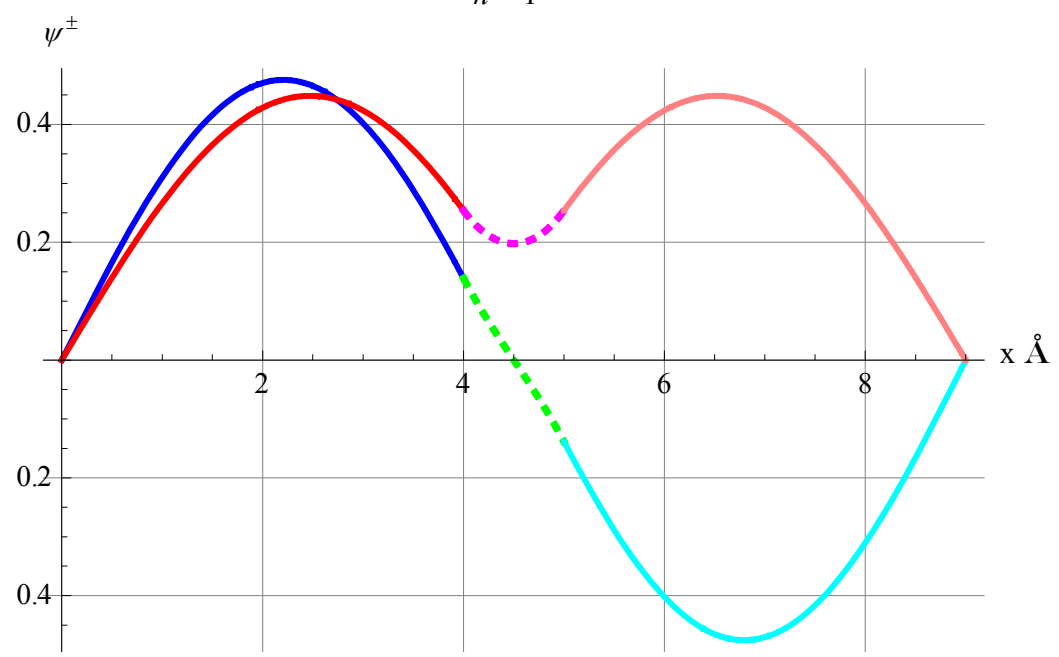

687

Copyright (C) 2011 SciRes.

JMP 
688

H. SARAFIAN
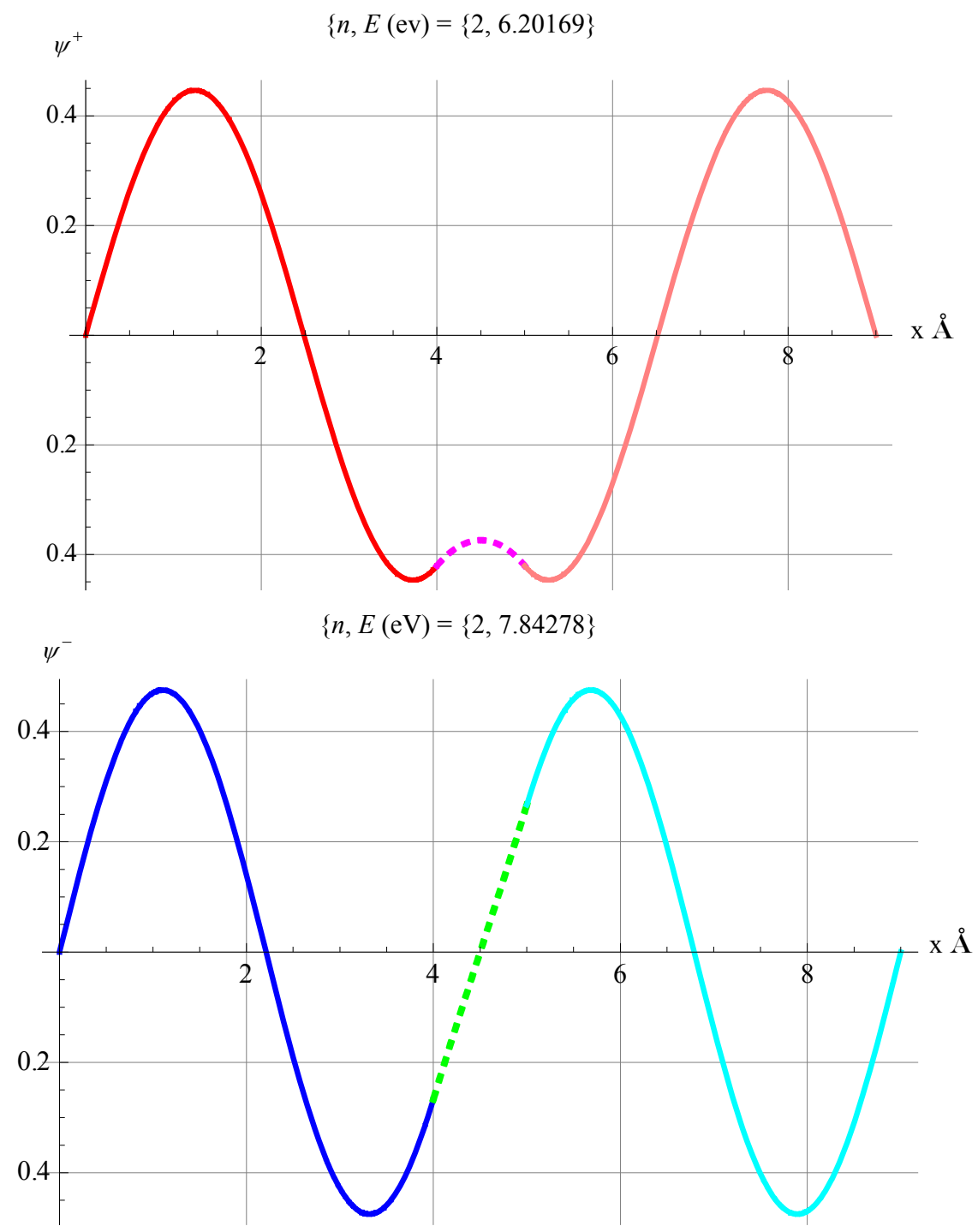

$n=2$

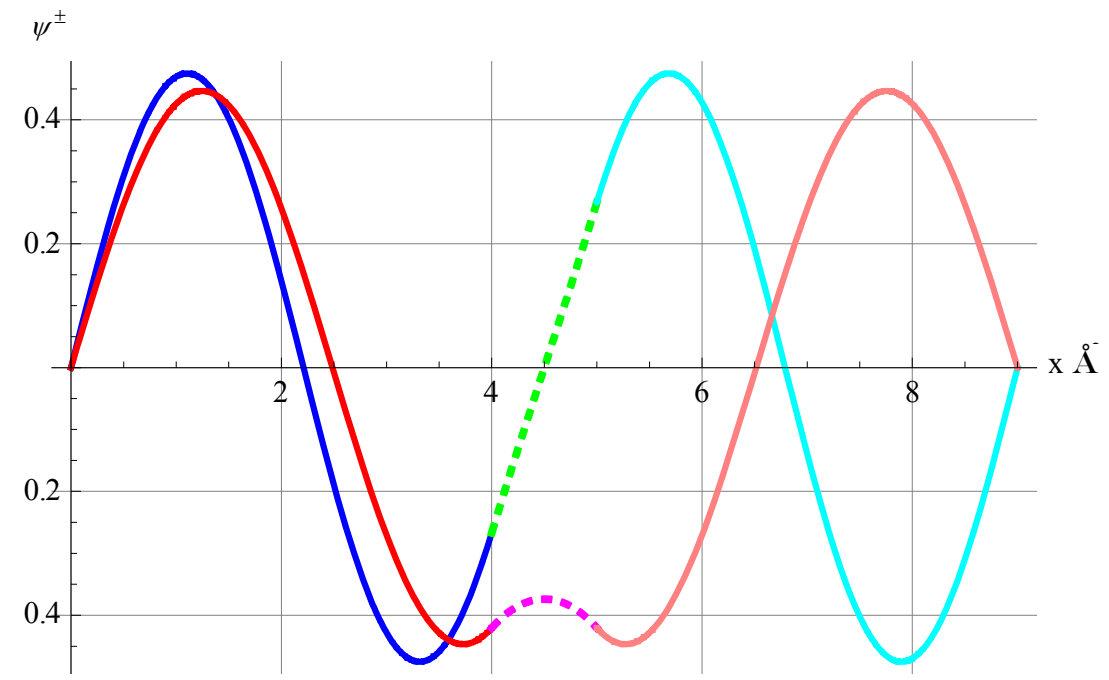

Figure 7. Description of these figures is the same as Figure 3. In this figure, the graphs are associated with $b=1.0 \AA$.

Copyright (C) 2011 SpiRes.

IMP 

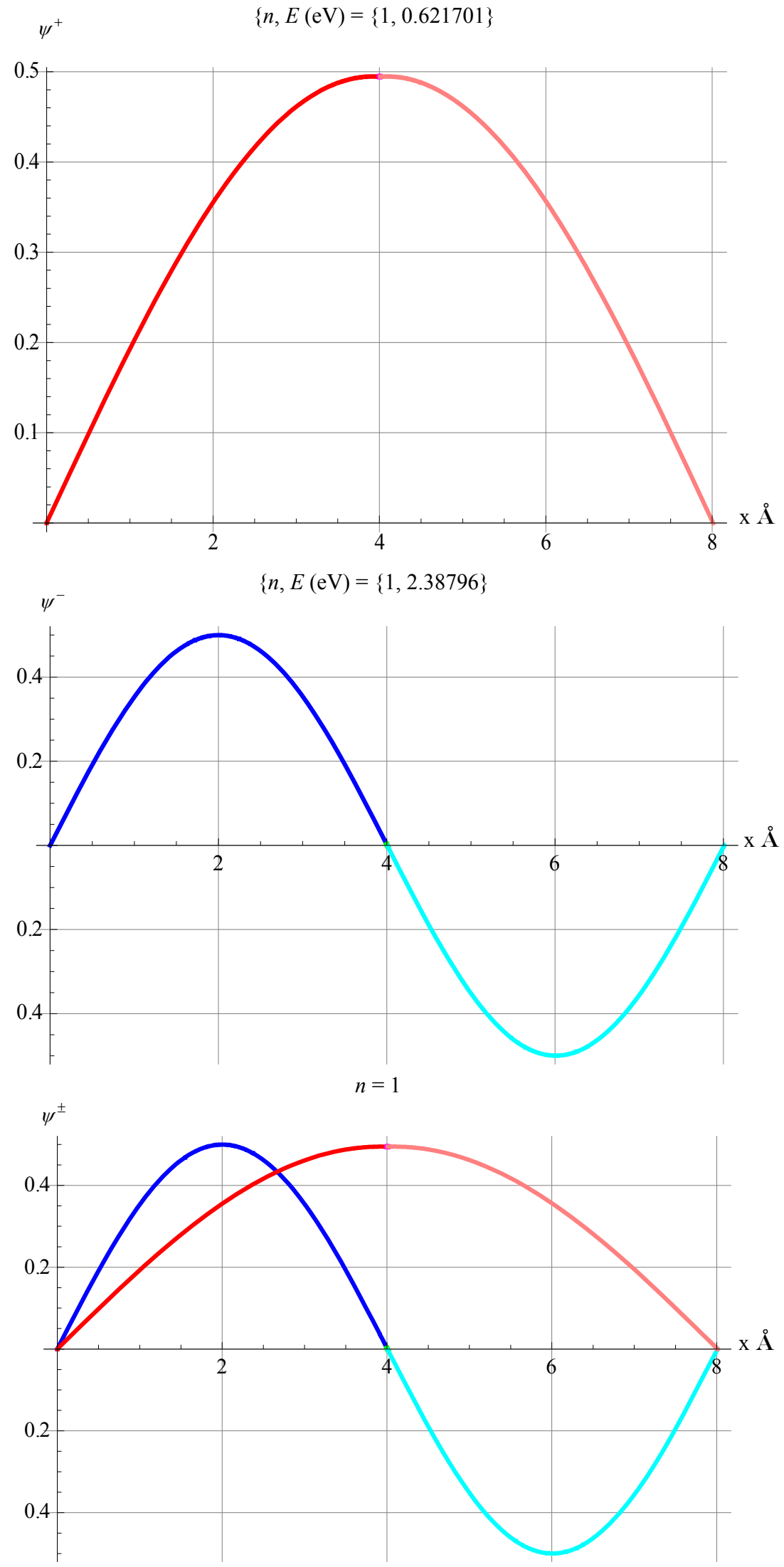
690

H. SARAFIAN
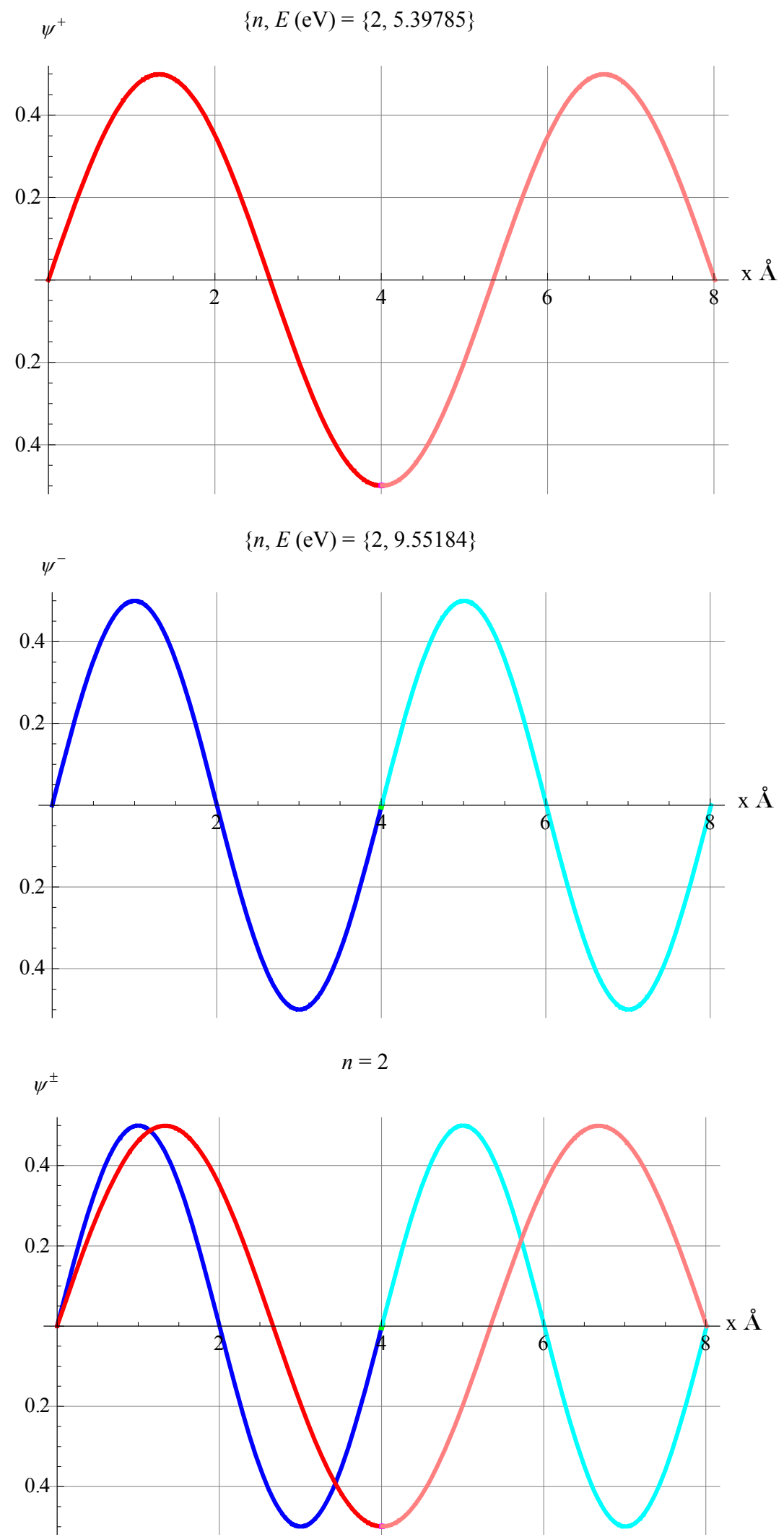

Figure 8. Description of these figures is the same as Figure 3. In this figure, the graphs are associated with $b=0.01 \AA$.

Copyright (C) 2011 SpiRes.

IMP 
Table 3. Description of this table is the same as Table 1. However, the geometric parameters of the potential are $\{a$, $b, c\}=\{4,2,6\}$.

\begin{tabular}{ccccccc}
\hline & Root 1 & Root 2 & Root 3 & Root 4 & Root 5 & Root 6 \\
\hline Plus & 0.869 & 2.393 & 2.393 & 5.386 & 5.386 & 6.777 \\
Minus & 0.598 & 1.775 & 3.448 & 6.649 & 6.649 & 7.702 \\
\hline
\end{tabular}

\subsection{Case 3, Symmetric and Asymmetric Negative Potentials}

In this subsection we extend our analysis considering a bound state electron within an attractive constant finite range potential. Similar to what we already discussed in the previous subsections we consider symmetrical as well as asymmetrical potentials.

For the sake of comparison we consider a double symmetrical potential well with geometric specifications $\{a, b$, $c\}=\{4,2,4\}, \AA$ and $\{V, h\}=\{-10,5\} \mathrm{eV}$, where $h$ is the height of the potential barrier from the bottom of the well. For the chosen scenario solving Equation (17) and/or
Equation (18) leads to only one permissible energy for the even and the odd wave functions. These wave functions along with the potential are shown in Figure 10.

Comparing these to their counterparts of positive energy barrier in Figure 3, one realizes: first, the number of allowed energies are reduced form two to one, and second, although the energies are quite different, their corresponding wave functions are somewhat similar. For the chosen specifications of the potential namely the $V$ and $h$ by varying the thickness $b$ we analyze its impact on the probability distribution. We also investigate the impact of the height of the potential $h$ on the probability distributions as well. These results are not reported here. However, it is worthwhile mentioning that by deepening the potential $V$ the number of the allowed bound states wave functions increase accordingly.

For the asymmetrical potential and for the sake of comparison between the positive potential case shown in Figure 9, we consider a potential with geometric char-
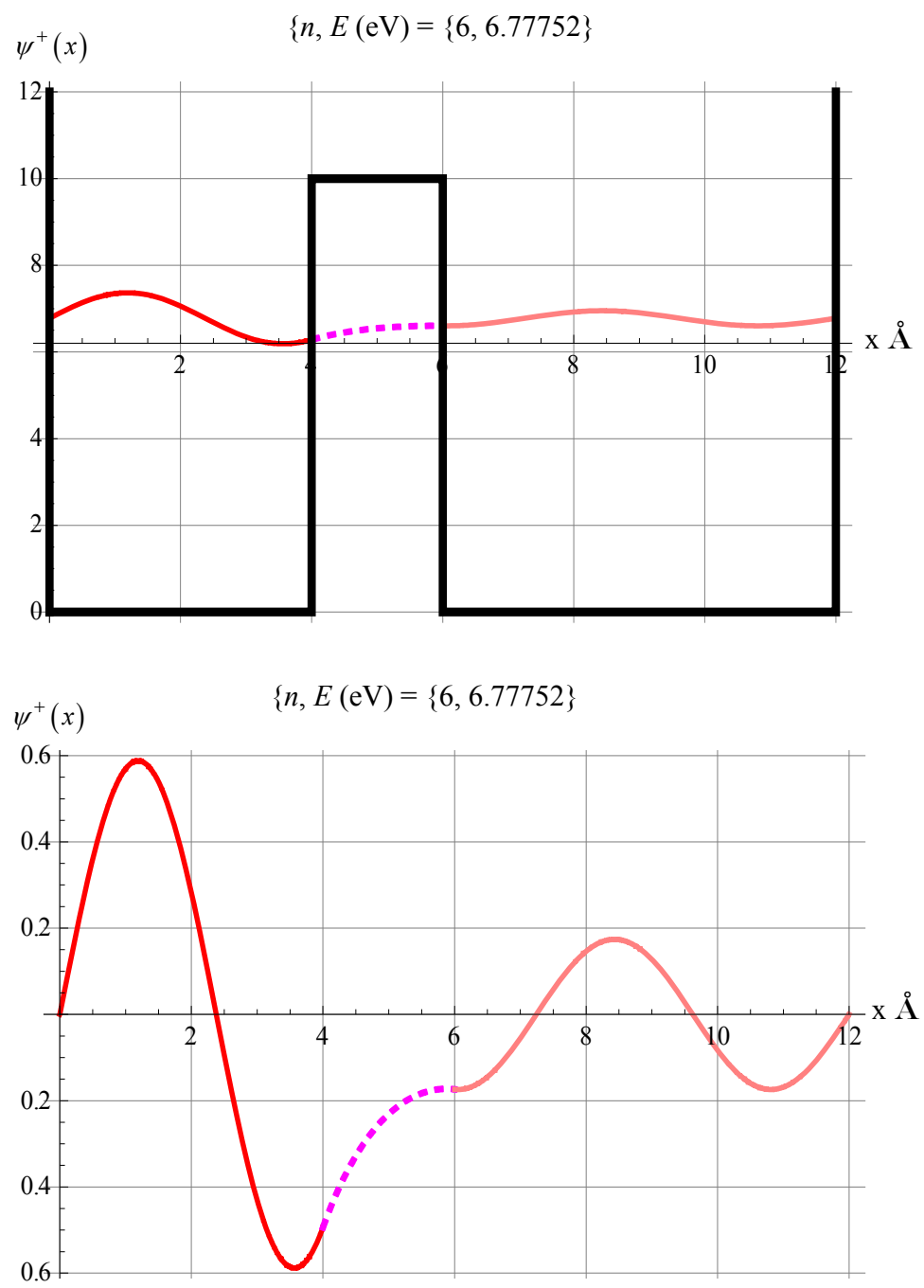
692

H. SARAFIAN
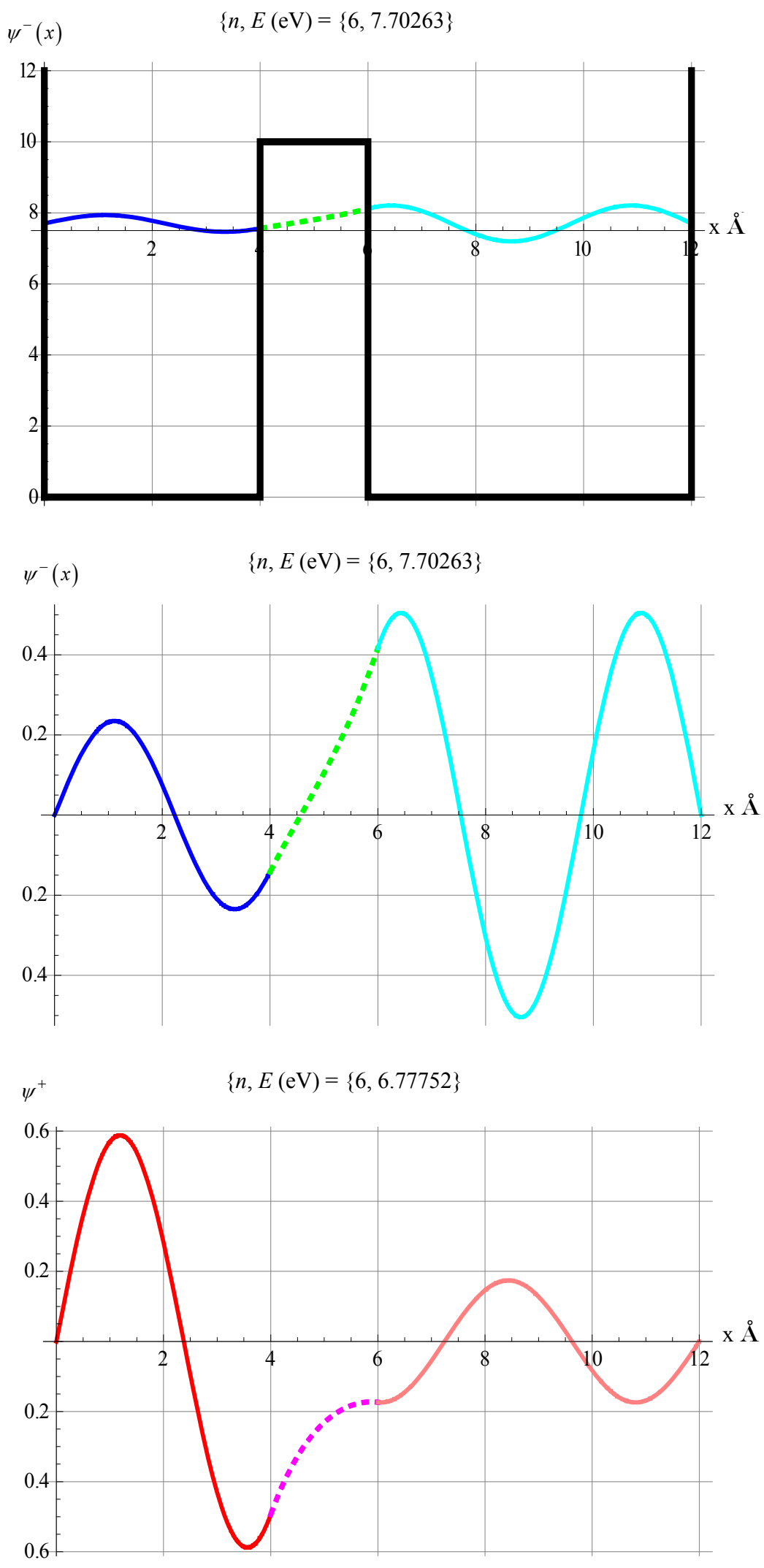

Copyright (C) 2011 SciRes.

JMP 

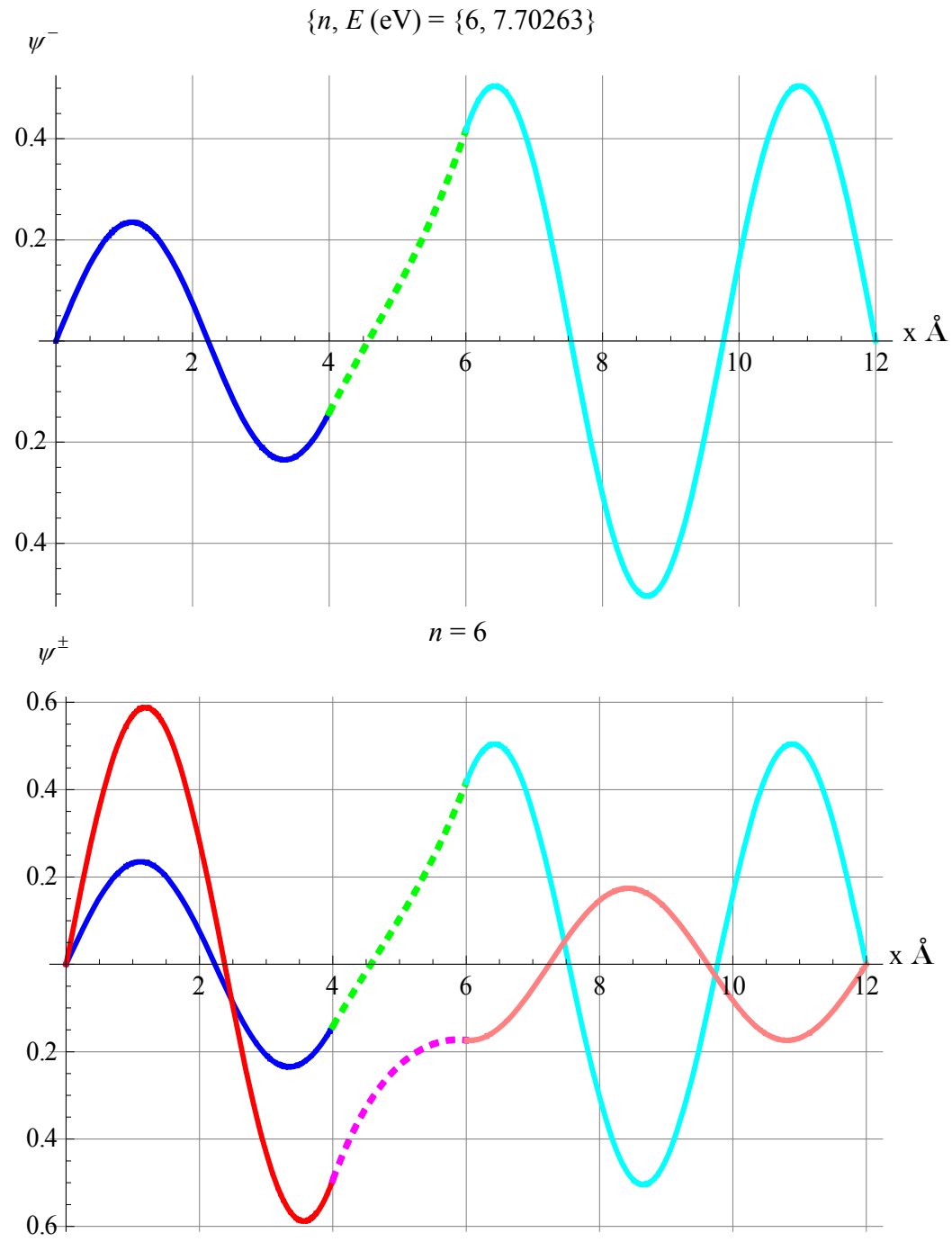

Figure 9. Description of these figures is the same as Figure 3 . The difference is $\{a, b, c\}=\{4,2,6\}$.

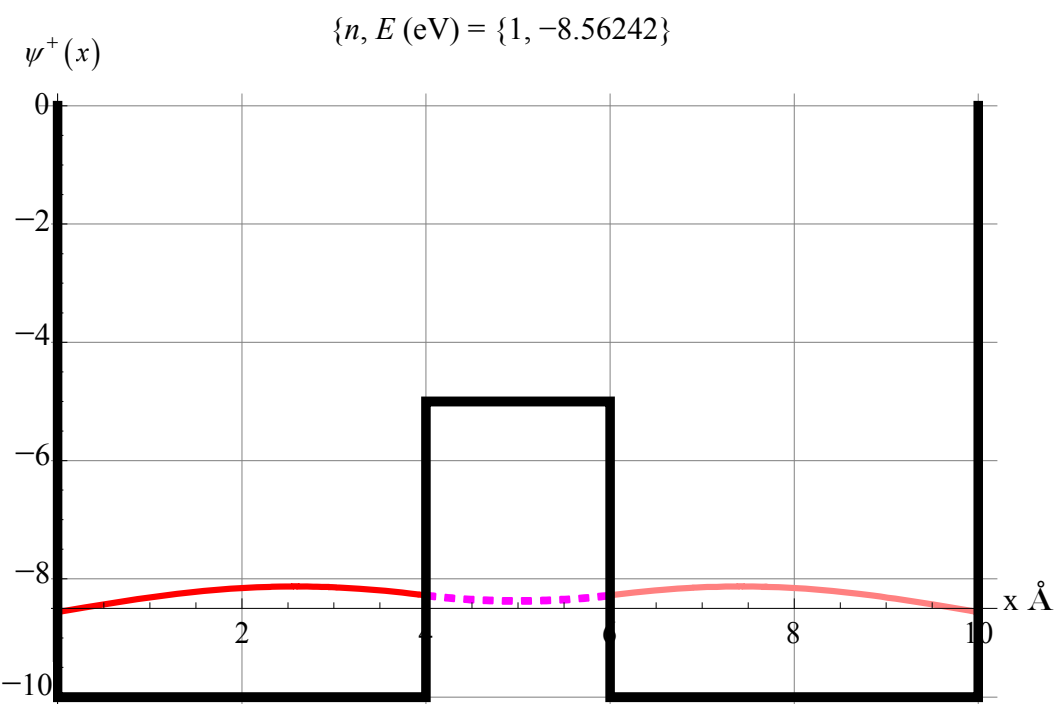



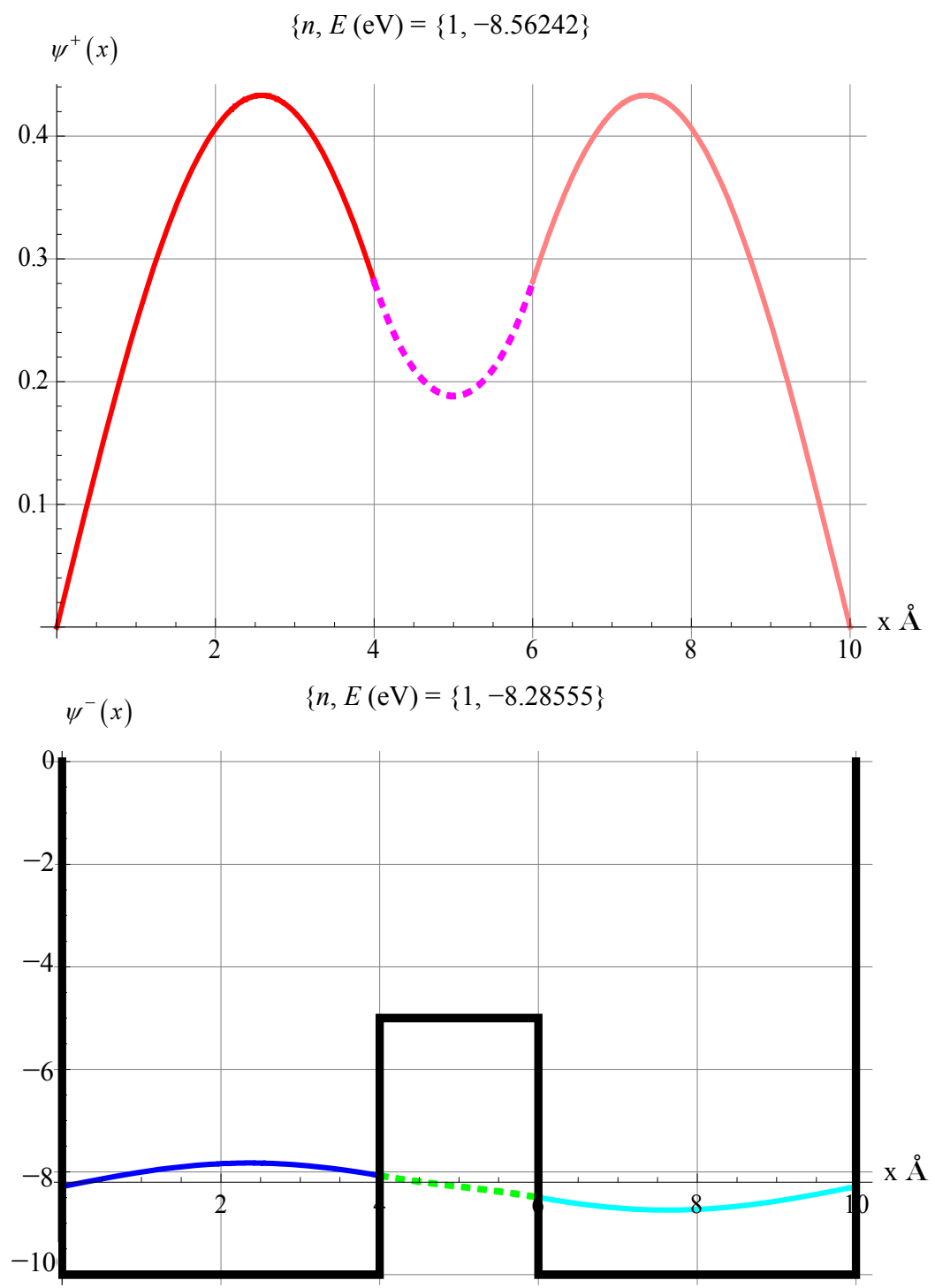

$\psi^{-}(x)$

$\{n, E(\mathrm{eV})=\{1,-8.28555\}$

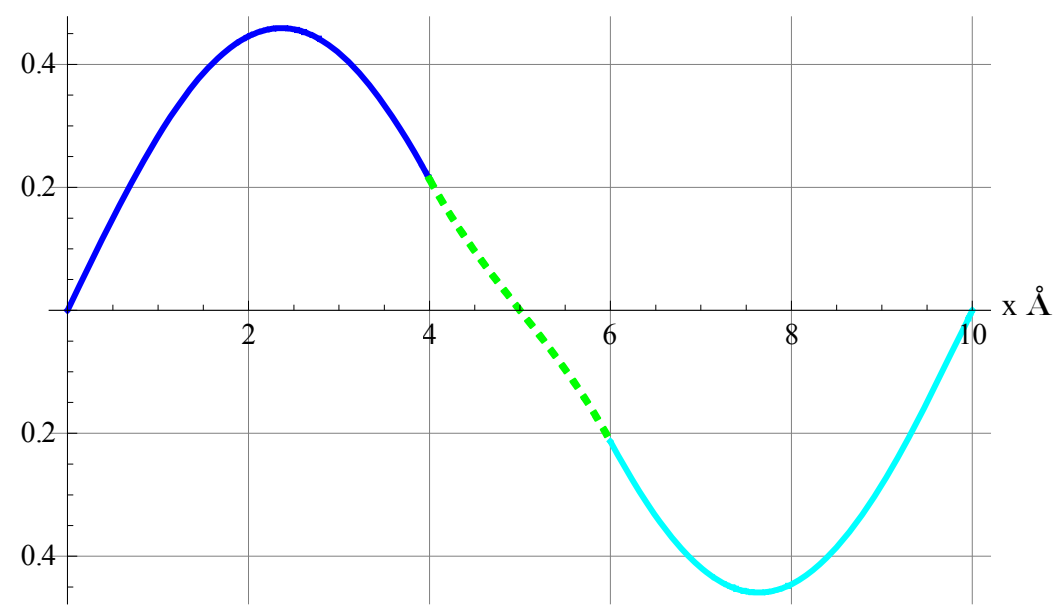



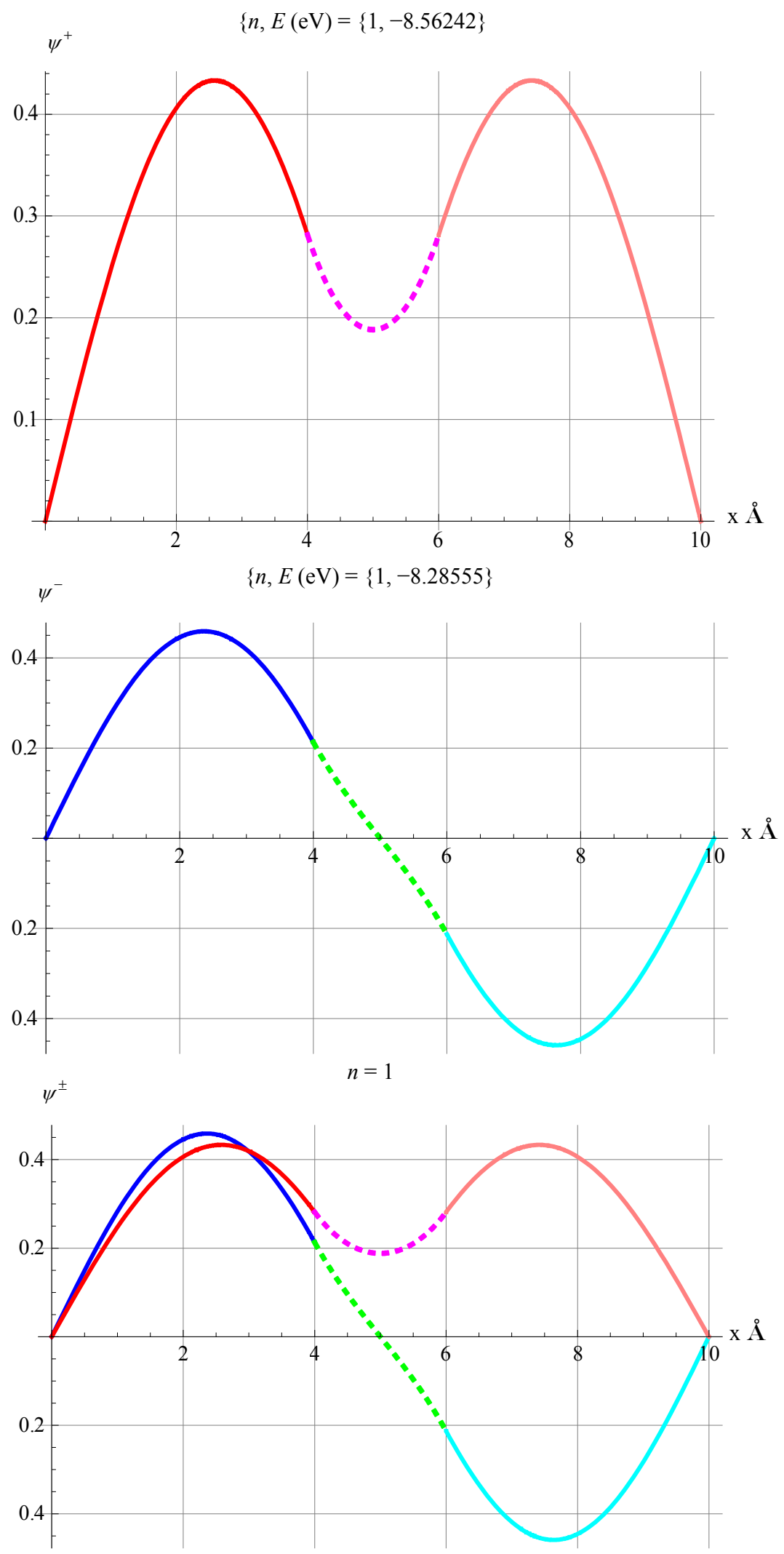

Figure 10. Description of these figures is the same as Figure 3 . The differences are, $V<0$ and $h=5 \mathrm{eV}$. 
acteristic $\{\mathrm{a}, \mathrm{b}, \mathrm{c}\}=\{4,2,6\} \quad \stackrel{0}{A}$. We also apply $\{V, h\}$ $=\{-10,5\} \mathrm{eV}$. According to our result the asymmetry of thepotential not only breaks the symmetry of the wave functions but it also changes the pairing of the associated allowed energies. For the case under consideration the number of the allowed energies for the even wave functions is three, where for the odd wave functions is four. We had no such situation for the positive asymmetrical potential. For the sake of visual understanding in Figure 11 we display the wave functions along with the potential for the lowest allowed energy.

Here again the author patiently exhausted analyzing the sensitivity of the wave functions and their associated probability distributions by varying parameters such as the thickness of the potential wall, $b$, and the height of the potential $h$. These all are compiled in a massive atlas.

\section{Conclusions}

Quantum tunneling is a curious phenomenon. It has no equivalent footing in classical physics. In the micro world it is assumed that particles with energies less than the potential are allowed to penetrate in the potential barrier gaining complex speed. Aside from trying to comprehend the phenomenon its detailed analysis inter twines with mathematical challenges. In current literature tunneling has been considered in one dimensional space with a given scenario such as free waves to describe the
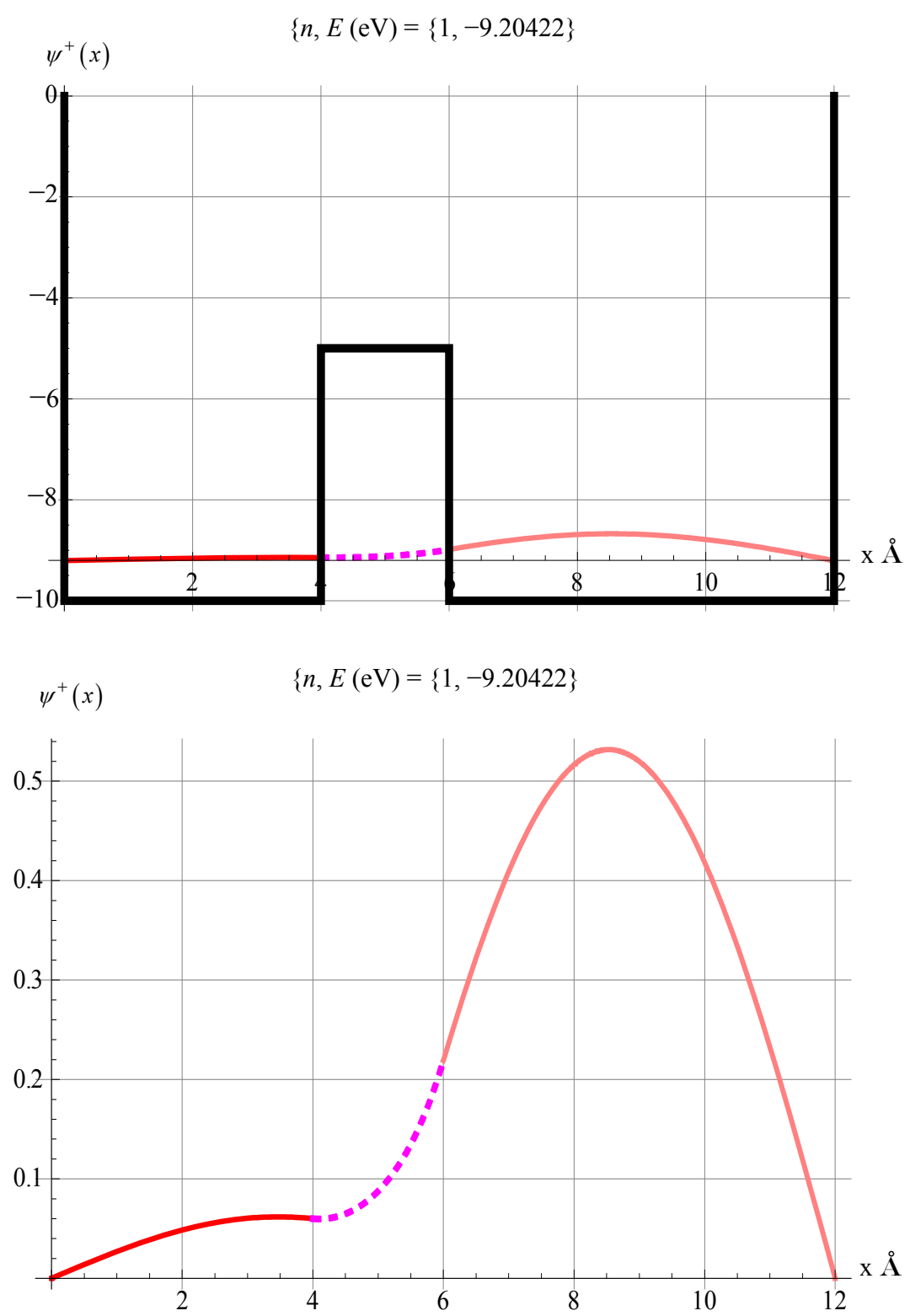

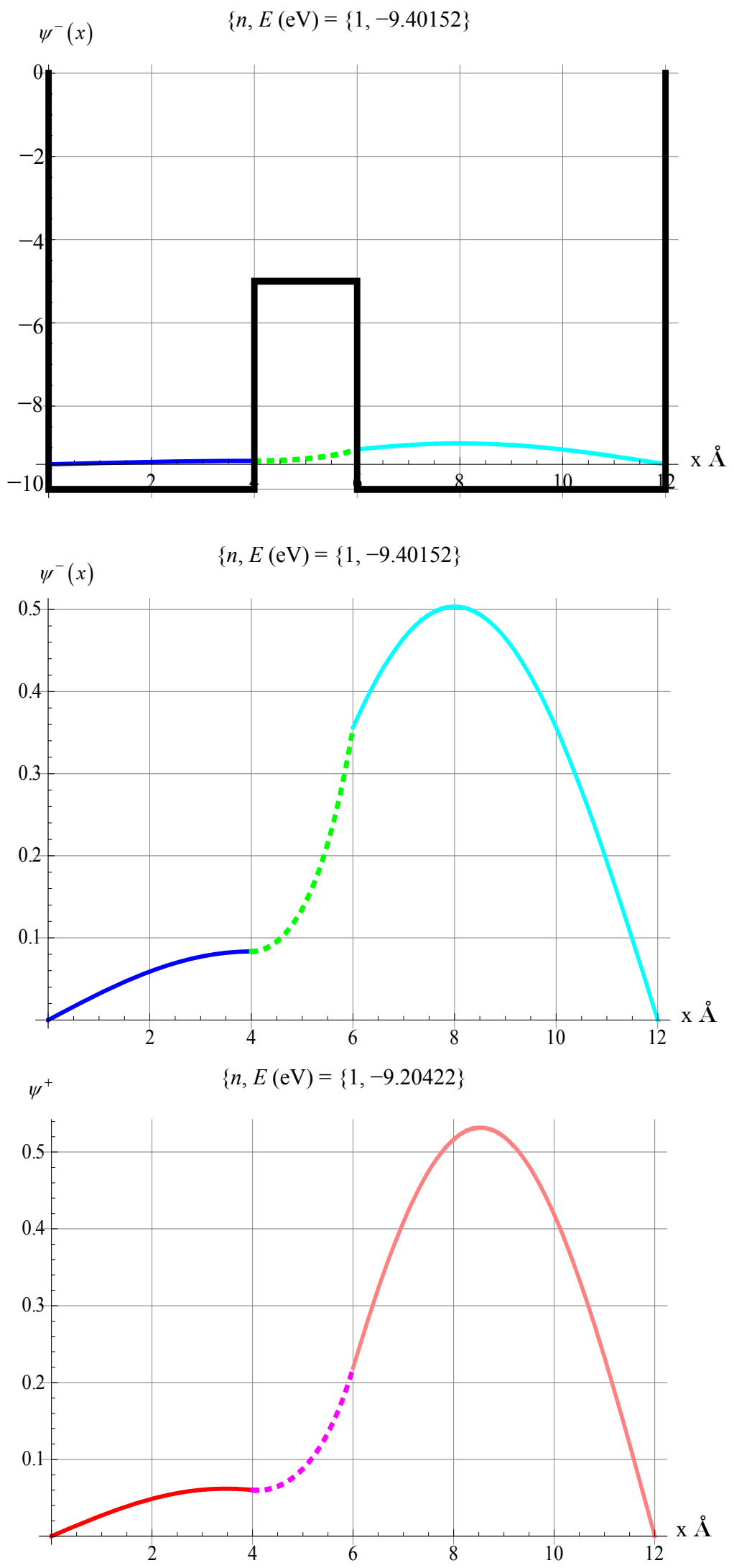

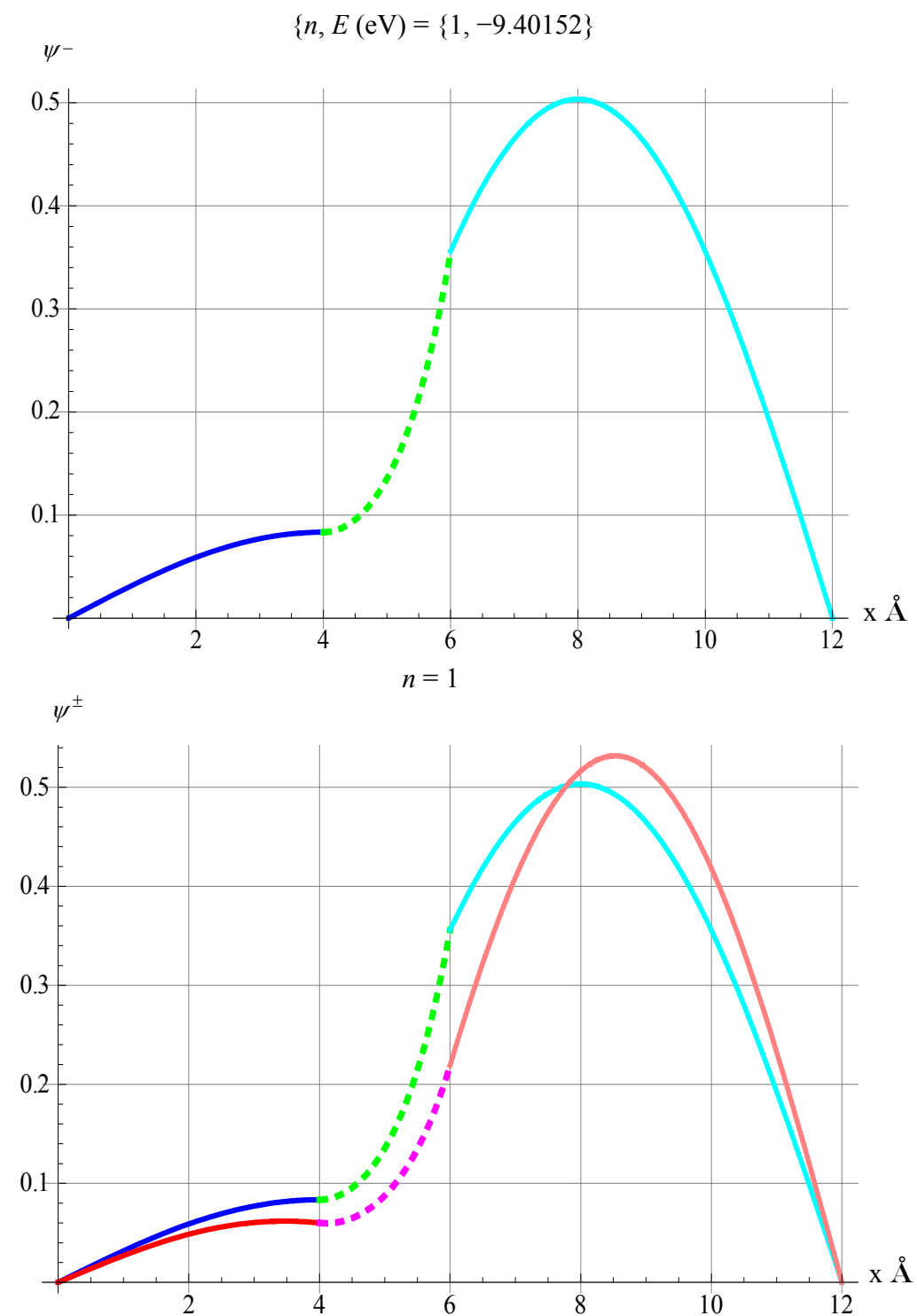

Figure 11. Description of these figures is the same as Figure 10. The difference is $\{a, b, c\}=\{4,2,6\}$.

initial and final states of a particle. In our analysis we consider different initial and final states revealing fresh features in conjunction with tunneling. Also by deploying a Computer Algebra System (CAS), such as Mathematica we analyze the problem beyond its traditional limits. From a CAS point of view composing a single file containing the needed various numeric and graphic modules is an advantage especially when one needs to test the "what-if" scenarios. The most labor intensive analysis such as searching for the sensitivity of the wave functions with respect to the thickness of the potential wall, the height and the depth of the potential has been compiled at ease. In addition, having the analytic formulation of the problem helps to form an informed opinion about the mathematical issues of quantum tunneling.

\section{Acknowledgements}

The author would like to thank Mrs. Nenette Hickey for carefully reading over the manuscript and making useful editorial comments.

\section{References}

[1] R. W. Gurney and E. U. Condon, "Quantum Mechanics and Radioactive Disintegration," Physical Review, Vol. 33, No. 2, 1929, pp. 127-140. doi:10.1103/PhysRev.33.127

[2] L. Schiff, "Quantum Mechanics," McGraw-Hill Company, Boston, 1968.

[3] G. Baym, "Lectures on Quantum Mechanics," W. A. 
Benjamin, Inc., New York, 1976.

[4] A. S. Davydov, "Quantum Mechanics," 2nd Edition, Pergamon Pr., Mesland, 1976.

[5] E. Merzbacher, "Quantum Mechanics," 3rd Edition, Wiley, Hoboken, 1997.

[6] http://www.youtube/QM5.1,QM5.4,QM6.1 and QM6.3

[7] J. M. Feagin, "Quantum Methods with Mathematica," Springer-Verlag, Berlin, 1994.
[8] Wolfram demonstration Projects, April 2011. http://demonstrations.wolfram.com.

[9] S. Wolfram, "The Mathematica Book," 4th Edition, Cambridge University Press, Cambridge, 1999 and "Mathematica"

[10] N. J. Giordano and H. Nakanishi, "Computational Physics," 2nd Edition, Pearson, Prentice Hall, Upper Saddle River, 2006. 